\title{
Resonant laser power build-up in ALPS—A "light shining through a wall" experiment
}

\author{
Klaus Ehret ${ }^{a}$, Maik Frede ${ }^{b}$, Samvel Ghazaryan ${ }^{a}$, Matthias Hildebrandt ${ }^{b}$, Ernst-Axel Knabbe ${ }^{a}$, \\ Dietmar Kracht ${ }^{\mathrm{b}}$, Axel Lindner ${ }^{\mathrm{a}, *}$, Jenny List ${ }^{\mathrm{a}}$, Tobias Meier ${ }^{\mathrm{c}}$, Niels Meyer ${ }^{\mathrm{a}}$, Dieter Notz ${ }^{\mathrm{a}}$, \\ Javier Redondo ${ }^{a}$, Andreas Ringwald ${ }^{a}$, Günter Wiedemann ${ }^{d}$, Benno Willke ${ }^{c}$ \\ a Deutsches Elektronen-Synchrotron DESY, Notkestraße 85, D-22607 Hamburg, Germany \\ ${ }^{\mathrm{b}}$ Laser Zentrum Hannover e.V., Hollerithallee 8, D-30419 Hannover, Germany \\ ${ }^{\mathrm{c}}$ Max-Planck-Institute for Gravitational Physics, Albert-Einstein-Institute, and Institut für Gravitationsphysik, Leibniz Universität, Hannover, Callinstraße 38, \\ D-30167 Hannover, Germany \\ ${ }^{\mathrm{d}}$ Hamburger Sternwarte, Gojenbergsweg 112, D-21029 Hamburg, Germany
}

\section{A R T I C L E I N F O}

\section{Article history:}

Received 27 May 2009

Received in revised form 19 October 2009

Accepted 20 October 2009

Available online 27 October 2009

Keywords:

Experimental tests

Photon regeneration

Resonators and cavities

Axions

Other gauge bosons

\begin{abstract}
A B S T R A C T
The ALPS Collaboration runs a "light shining through a wall" (LSW) experiment to search for photon oscillations into "weakly interacting sub-eV particles" (WISPs) inside of a superconducting HERA dipole magnet at the site of DESY. In this paper we report on the first successful integration of a large-scale optical resonant cavity to boost the available power for WISP production in this type of experiments. The key elements are a frequency tunable narrow line-width continuous wave laser acting as the primary light source and an electronic feed-back control loop to stabilize the power build-up. We describe and characterize our apparatus and demonstrate the data analysis procedures on the basis of a brief exemplary run.
\end{abstract}

(c) 2009 Elsevier B.V. All rights reserved.

\section{Introduction}

The Standard Model (SM) of elementary particles offers an accurate description of almost every phenomenon observed so far in particle physics. Despite this success, there are both theoretical and observational motivations to believe what the SM describes is just a small component of nature's complexity. From the theoretical side, the SM suffers from naturalness, hierarchy and arbitrariness problems and, moreover, it does not describe gravity. From the observational viewpoint, cosmology and astrophysics claim that around $22 \%$ of the universe's energy density is made of a yet unidentified type of non-baryonic "dark matter" and $74 \%$ of a yet more mysterious "dark energy".

Extensions of the SM usually introduce many new particles and symmetries that provide elegant solutions to our theoretical concerns and candidates for the "dark sector". Interestingly enough, these extensions often include many more particles than known today. On general grounds these particles could exist at any energy scale since new symmetries could protect their masses.

\footnotetext{
* Corresponding author.

E-mail address: axel.lindner@desy.de (A. Lindner).
}

In particular, nothing prohibits that light particles beyond the SM exist as long as they have no SM charges, i.e. as long as they populate a "hidden sector". Actually, these "hidden sectors" arise quite naturally in string theory, our current best candidate for a theory of quantum gravity, and they are required to break the hypothetical supersymmetry-a higher symmetry proposed to solve the hierarchy problem. Interestingly, broken supersymmetry provides good dark matter candidates, the so-called WIMPs (for weakly interacting massive particles).

Low mass particles living in "hidden sectors" might still have very weak interactions with the SM fields through radiative corrections involving massive "mediator" particles or through gravity. We name these particles WISPs (weakly ${ }^{1}$ interacting sub$\mathrm{eV}$ particles). The feebleness of their interactions implies extremely few events in a typical experiment, making the luminosity and detector efficiency two crucial requirements for WISP searches. On the other hand, having low masses, WISPs can potentially exhibit coherent interactions along macroscopic distances boosting their production probabilities.

\footnotetext{
${ }^{1}$ Here "weak" has to be understood in a broad sense, while in WIMP, it stands for electro-weak.
} 
A laser beam can provide both high luminosity and very good coherence properties. Commercial lasers in the visible spectrum can easily reach output powers of several tens of Watts $\left(~ \gtrsim 10^{19}\right.$ photons/s) and can have coherence lengths of several kilometers. Sensitive detectors with quantum efficiencies close $\sim 100 \%$ are also available. In this sense, experimental searches for WISPs coupled to photons seem very favored.

Let us notice that these WISPs can have a sizable impact on cosmology and astrophysics. Often not realized, they can be as good cold dark matter candidates as WIMPs despite having sub-eV masses, as it is the case for the axion [1]. Stellar evolution is often accelerated by the emission of WISPs [2-5], as it is by neutrinos, and photon-WISP interactions can modify the spectra of cosmic radiation [6-11]. Indeed, WISPs could help in understanding a number of recent observations [11-18]. Strong constraints on WISPs often arise from these effects [19], but they are certainly model dependent [20-24] and they rely on our very imperfect knowledge of cosmology, stellar interiors or astrophysical environments.

A very spectacular effect of such WISPs takes place when we have a photon-WISP interaction vertex in our theory, a so-called mixing term. In this context, photons can convert into WISPs during their propagation, and the quantum amplitudes of these transitions at different points along the trajectory can add up coherently, enhancing the signal, or decreasing it if they add up out of phase. This phenomenon is known as quantum flavor oscillations and has been observed in the context of the neutral kaon, B meson and neutrino systems.

There are several direct and indirect experimental approaches for WISP searches [5,25-42]. Possibly, the cleanest way to search for photon-WISP oscillations is through so-called "light shining through a wall" (LSW) experiments [43-45]. In such experiments a laser beam is shone onto a thick wall where photons are stopped but WISPs produced in oscillations can traverse. By the inverse process, WISPs can re-oscillate into photons behind the wall and may be detected in a low background environment, see Fig. 1.

Since the number of photons regenerated after the wall is proportional to the flux in the "oscillation" region, it is crucial to get the highest possible laser powers. In this respect, it is extremely disappointing that photons in the oscillation region are lost when hitting the wall. To partially overcome this situation, in a pioneer LSW experiment $[46,47]$ a so-called optical delay line was set up which forced the light to travel on almost collinear paths for $\approx 200$ times through the oscillation region, thus augmenting the effective laser power by a factor $\approx 100$. In trying to go much further than this figure, one might suffer, however, from two drawbacks. The first is the necessity of a detector with a large sensitive area because the different beam paths must not overlap in order to avoid destructive interference. However, in general the dark noise of a detector for single photons grows with the size of its sensitive area. Thus it will increase with an increasing number of light passes in the delay line and by this deteriorating the signal to noise ratio of the detector. The second drawback is that delay lines with a much higher number of light passes than 200 are difficult to construct due to the growing complexity of the underlying geometrics.



Fig. 1. Schematic overview of a "light shining through a wall experiment". The gray blob indicates the mixing term between photons and the "weakly interacting sub-eV particle" (WISP).
These problems can be solved to some extent by the use of optical resonators. Since they are based on the superimposition of all the different equivalent roundtrips within the area of only one light path, the sensitive area of the detector can be kept very small resulting in low dark noise. On the other hand, long optical cavities with equivalent round trip numbers of several thousand are used by some large-scale experiments such that they boost up the power inside to levels of several tens of kW. Good examples of these are the laser interferometric gravitational wave detectors like GEO600 [48] or LIGO [49].

Due to this power enhancement, resonant optical cavities could be extremely useful also in a LSW experiment where they would act as amplifiers for the photon flux that is available from the laser source [50-52]. However, such a set-up was never realized so far. $^{2}$ A likely reason for that is the by far increased complexity of a cavity-enhanced LSW-scheme. This complexity arises from strict constraints on the experimental ambient conditions such as high cleanliness or large vacuum chambers $[48,49,56]$ and the necessity, in some important physics cases, to install the cavity in a strong magnetic background field.

In this paper we report on the first successful realization of such a cavity-enhanced LSW experiment, the "Any-Light-ParticleSearch" (ALPS) experiment [57,58] at DESY in Hamburg. It utilizes a long optical resonator on the experiment's production side inside a superconducting HERA dipole. The resonator increases the effective laser power for WISP searches by more than a factor of 40. Its suitability as part of a large-scale LSW experiment is demonstrated by a first search for WISPs at ALPS. The current limitations for its sensitivity enhancement together with other possible improvements of the experiment are identified. This should enable us to increase the sensitivity of the whole experiment significantly in the future.

This paper is organized as follows: In Section 2 we briefly describe the WISPs we can search for in the ALPS experiment and characterize the phenomenon of oscillations. In Section 3 we detail the components of the ALPS experiment. The design and characterization of the optical cavity are described in Section 4 while in Section 5 we explain the process of data taking, review the performance of the optical resonator and the detector during the measurement run and present the results.

\section{Photon-WISP oscillations and the WISP zoo}

The equations of motion of the photon-WISP system as a function of length can be written as

$i \frac{d}{d L}\left(\begin{array}{l}|\gamma\rangle \\ |\phi\rangle\end{array}\right)=\frac{1}{2 \omega}\left(\begin{array}{cc}-2 \omega^{2} \Delta n & \delta \\ \delta & m_{\phi}^{2}\end{array}\right)\left(\begin{array}{l}|\gamma\rangle \\ |\phi\rangle\end{array}\right)$

where $\omega$ is the photon energy, $\Delta n=n-1$ with $n$ the photon refraction index in the medium, $m_{\phi}$ is the WISP mass and $\delta$ is the quantum mechanical amplitude of the $\gamma \rightarrow \phi$ forward transition $\delta=\left\langle\phi\left|\mathcal{H}_{\text {int }}\right| \gamma\right\rangle$.

The transition probability shows the characteristic oscillatory behavior as a function of the distance $L$

$P(\gamma \rightarrow \phi)=\frac{4 \delta^{2}}{M^{4}} \sin ^{2} \frac{M^{2} L}{4 \omega}$

with $M^{2}=\left(\left(2 \omega^{2} \Delta n+m_{\phi}^{2}\right)^{2}+4 \delta^{2}\right)^{1 / 2}$. Unfortunately, for visible light one usually has $\Delta n>0$ so one cannot match $2 \omega^{2} \Delta n+$ $m_{\phi}^{2}=0$ by choosing a suitable medium to maximize the amplitude

\footnotetext{
${ }^{2}$ Cavities with excellent characteristics have been combined with strong magnetic fields in other types of WISP searches, like in the PVLAS $[53,54]$ or Q\&A [55] searches of WISP-induced dichroism and birefringence.
} 


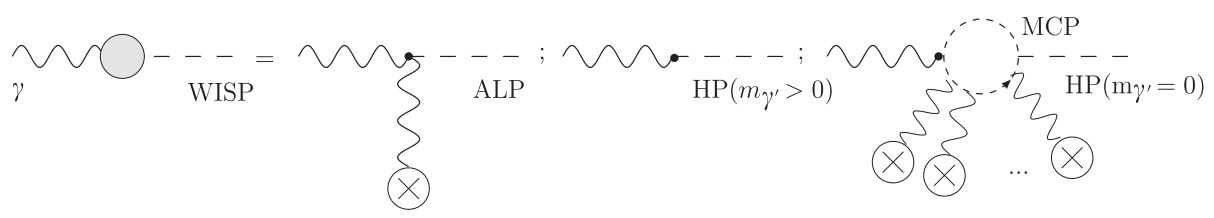

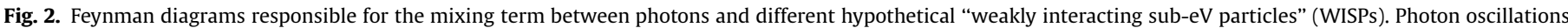

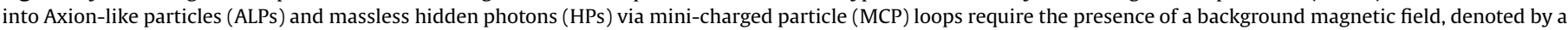
crossed circle. Oscillations into massive Hidden Photons occur regardless of such a background. See Table 1 for details on the photon-WISP couplings and references.

of the oscillations. Given an experimental set-up, i.e. $L$ and $\omega$, there is a critical value for $M, M_{\text {crit }}=\sqrt{2 \pi \omega / L}$, above which the probability can be strongly suppressed if $m_{\phi}^{2} \ll 2 \omega^{2} \Delta n$. On the other hand, if we are in coherent conditions $\left(M \ll M_{\text {crit }}\right)$ the probability becomes independent of $m_{\phi}$ and $\Delta n$, taking a simpler form

$P(\gamma \rightarrow \phi)=\delta^{2} L^{2} /\left(4 \omega^{2}\right)$

where the coherent enhancement in the interaction length $L$ is evident. Therefore, without an a priori knowledge of the WISP mass, we can optimize the mass coverage of the experiment if the oscillation region is in vacuum $(\Delta n=0)$, which for practical purposes means keeping $2 \omega^{2} \Delta n$ smaller than $M_{\text {crit }}$.

Lorentz invariance forbids $\gamma \rightarrow \phi$ transitions when the WISP spin is different from 1 , and has to be explicitly broken if we want to prove oscillations into spin-0 (or $>1$ ) particles. In the ALPS setup this is done by the inclusion of a strong magnetic field $B^{\text {ext }}$ orthogonal to the propagation direction. In this case, photons polarized along the magnetic field or perpendicular to it can (and will) behave differently. They will have different $\Delta n$ and $\delta$ so their WISP oscillation probability will be generically different.

The first WISPs to be considered are axions and axion-likeparticles (ALPs). They are well motivated spin-0 particles that couple to two photons depending on their intrinsic parity via interaction terms in the Lagrangian such as

$\mathcal{L}_{\text {int }}^{-}=g_{-} \phi E \cdot B \quad$ and $/$ or $\quad \mathcal{L}_{\text {int }}^{+}=g_{+} \phi \frac{1}{2}\left(E^{2}-B^{2}\right)$

where $g_{ \pm}$are dimensionful coupling constants, $\phi$ the ALP field and $E, B$ the electric and magnetic fields. In models where parity is nonconserved [59] both couplings are allowed. Such schizons have very similar phenomenology to the ALPs studied here [60,61].

Parity odd ALPs $\left(0^{-}\right)$arise as Nambu-Goldstone bosons [62-64] (GB) of spontaneously broken global axial symmetries, with the QCD axion [65-74] the most famous but not unique example [75-82]. Parity even ALPs $\left(0^{+}\right)$can be GBs, but also quintessence fields [83-86] or fields governing the sizes of extra dimensions (moduli) or gauge couplings (dilatons) in string theories. Such particles are in principle subject to strong constraints from deviations of Newton's law [87], although some models which evade astrophysics also overcome these problems [21,24].

Further WISP candidates are hidden photons (HPs) [43]. They are gauge bosons of $\mathrm{U}(1)_{\mathrm{h}}$ symmetries of a hidden sector, which are ubiquitous in extensions of the Standard Model, e.g. Refs. [88-91]. Gauge invariance allows HPs to have a Stückelberg mass $[92,93]$ and kinetic mixing with the standard photon $[94,95]$

$\mathcal{L}_{\text {mix }}=\frac{1}{2} \chi F_{\mu v} B^{\mu v}$

with $F^{\mu v}\left(B^{\mu v}\right)$ the photon (HP) field strength tensors and $\chi$ a dimensionless coupling ranging typically from $\sim 10^{-23}$ to $\sim 10^{-2}$ [96-99]. Models with kinetic mixing have a wide range of phenomenological applications, e.g. Refs. [100-110].

As a final example, hidden sector particles charged under $\mathrm{U}(1)_{\mathrm{h}}$, get a non-zero electric charge through the kinetic
Table 1

Parameters characterizing the photon-WISP probability of oscillations Eq. (3)

\begin{tabular}{llllll}
\hline WISP & Needs & $\delta_{\|}$ & $\delta_{\perp}$ & $m_{\phi}^{2}$ & Ref. \\
& $B^{\text {ext }}$ & & & & \\
\hline Parity odd ALP $\left(0^{-}\right)$ & Yes & $g_{-} B^{\text {ext }} \omega$ & 0 & $m_{\phi_{-}}^{2}$ & {$[114]$} \\
Parity even ALP $\left(0^{+}\right)$ & Yes & 0 & $g_{+} B^{\text {ext }} \omega$ & $m_{\phi_{+}}^{2}$ & {$[115]$} \\
HP $\left(m_{\gamma^{\prime}}>0\right)$ & No & $\chi m_{\gamma^{\prime}}^{2}$ & $\chi m_{\gamma^{\prime}}^{2}$ & $m_{\gamma^{\prime}}^{2}$ & {$[43]$} \\
$\begin{array}{c}\text { MCP }+ \text { HP }(\text { for } \\
\left.m_{\gamma^{\prime}}=0\right)\end{array}$ & Yes & $-2 \chi \omega^{2} \Delta N_{\|}$ & $-2 \chi \omega^{2} \Delta N_{\perp}$ & $-2 \omega^{2} \Delta N_{\|, \perp}$ & {$[113]$} \\
\hline
\end{tabular}

The mixing parameter $\delta$ depends on the relative orientation of the photon polarization and the external field which can be parallel $\delta_{\|}$and perpendicular $\delta_{\perp}$. Note that in the MCP+HP case, the indices of refraction due to MCP loops $\Delta N_{\|, \perp}=\Delta N_{\|, \perp}\left(Q_{\mathrm{MCP}}, B^{\mathrm{ext}}\right)$ are generally complex and Eq. (3) does not hold. See Ref. [113] for the adequate expression.

mixing term [94]

$Q_{\mathrm{MCP}}=\frac{e_{\mathrm{h}}}{e} \chi$

where $e_{\mathrm{h}}$ is the gauge coupling of $\mathrm{U}(1)_{\mathrm{h}}$ and $e$ the electron's charge. Another MCP model beyond this paradigm can be found in Ref. [111]. Since $\chi$ is usually very small we call them mini-charged particles (MCPs). Photons propagating in external magnetic fields will pair produce MCPs [40,112] and MCP loops can produce photon $\rightarrow$ HP oscillations even if the HP mass is zero [113].

The Feynman diagrams giving rise to photon-WISP mixing in these different models are shown in Fig. 2 and the parameters characterizing the oscillation amplitudes in Table 1.

\section{The ALPS experiment}

The ALPS experiment as sketched in Fig. 3 is built up along a superconducting HERA dipole magnet which provides a field intensity of $B=5.30 \mathrm{~T}$ in a length of $8.8 \mathrm{~m}$. Two vacuum tubes are inserted into the magnet at each of both ends, featuring 6.3 and $7.6 \mathrm{~m}$ length, respectively. For both tubes the length in the magnetic field amounts to $4.3 \mathrm{~m}$. WISP production takes place in the first tube; regeneration of photons in the second one. Around the first tube we have arranged an optical resonator to increase the power available for WISP production. A light-tight plug is placed at the inside end of the second tube as our "wall" to absorb photons leaving the cavity towards the regeneration tube.

A continuous-wave emitting laser at $1064 \mathrm{~nm}$ wavelength is used as the light source. For detection reasons, this light is frequency doubled with a second harmonic generator before it enters the optical resonator that constitutes the production part. Regenerated photons are re-directed by an oblique mirror and focused by a lens into a few pixels of our CCD camera. The volume containing the hypothetical regenerated beam is sealed against environmental light entering into the signal region of the camera. Right at the entrance of the resonator a very small part is separated from the incident beam as a reference for the alignment 


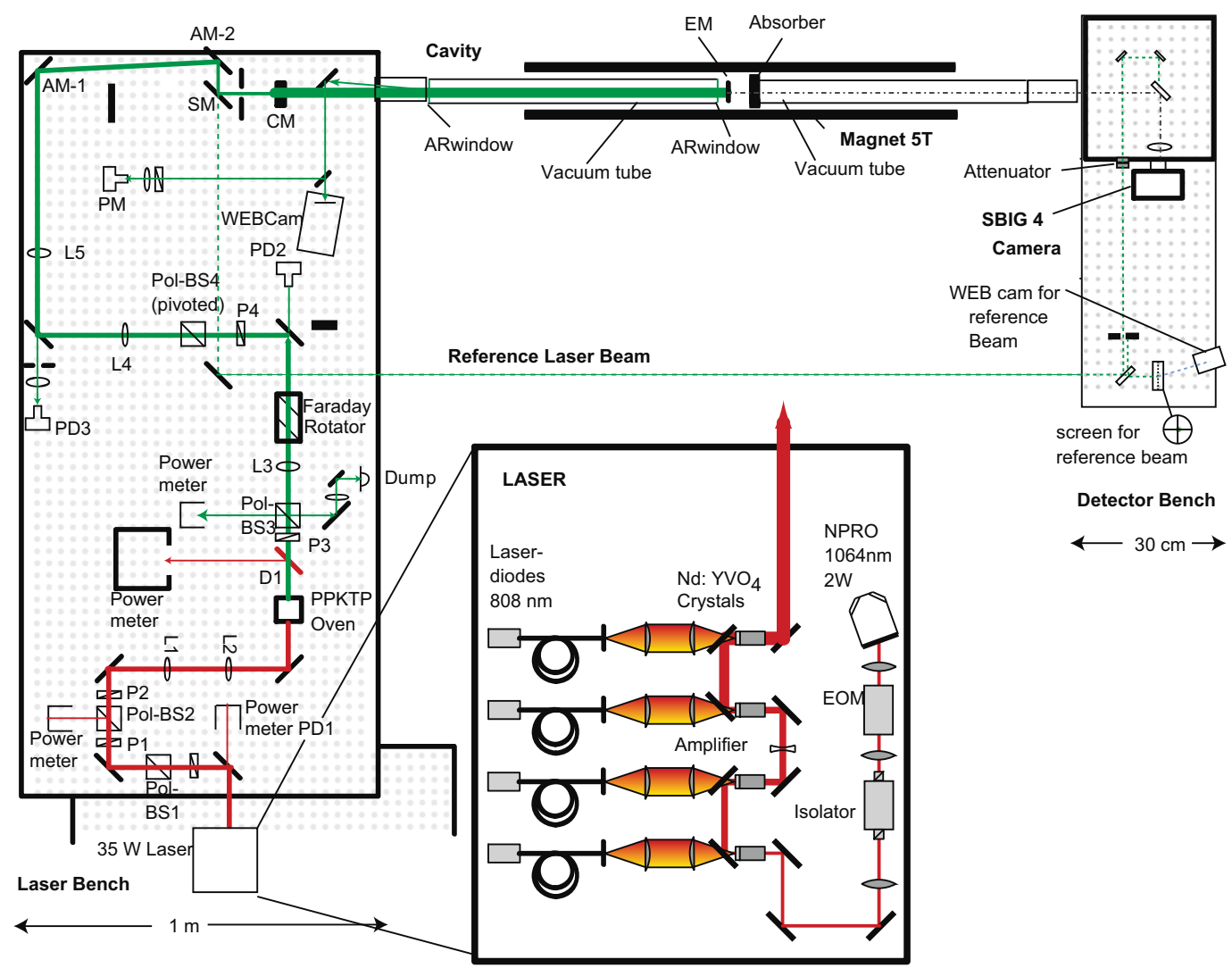

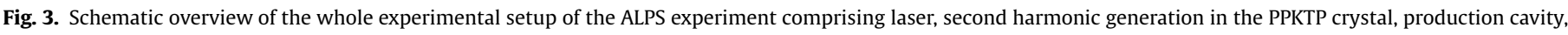
magnet and detector. Magnified is the schematic representation of the ALPS master-oscillator power amplifier laser system.

of the beam axis of the resonator. This reference beam is guided along the magnet's side from the laser bench to the detection bench. After very strong attenuation the reference beam is also focused by the same lens onto a different position of the CCD.

In the remainder of this section we present the most relevant characteristics of the different components of our setup.

\subsection{Laser}

The beam tube inside the HERA dipole magnet is bent horizontally and leaves an aperture of only $14 \mathrm{~mm}$ with the vacuum tubes installed. This strongly constrains the beam quality of a suitable laser [57]. Furthermore, to efficiently increase the optical power with a cavity, one needs a continuous-wave laser that emits a single longitudinal and transversal mode with a much smaller linewidth than the one of the resonator. Thus, the laser source used for the ALPS experiment is a narrow-linewidth master-oscillator power amplifier system (MOPA) operating at $1064 \mathrm{~nm}$ (Fig. 3). It is based on the system [116] developed for gravitational wave detectors like LIGO, GEO and VIRGO.

Stable narrow-linewidth emission is provided by a non-planar ring oscillator (NPRO) [117], emitting $2 \mathrm{~W}$ of output power with a spectral width of $1 \mathrm{kHz}$ (measured over $100 \mathrm{~ms}$ ) and a long term frequency stability of $1 \mathrm{MHz} / \mathrm{min}$. In four diode pumped $\mathrm{Nd}: \mathrm{YVO}_{4}$ amplifier stages the output power of this laser is increased to $35 \mathrm{~W}$ preserving the spectral emission characteristics and the nearly diffraction limited beam quality with a fundamental transverse mode content of $95 \%$. The MOPA is equipped with several frequency control elements, which are applied for the cavity frequency locking scheme in the ALPS experiment. A piezoelectric transducer installed on the NPRO laser crystal allows for a frequency shift of $\pm 100 \mathrm{MHz}$ with a response bandwidth of $100 \mathrm{kHz}$. Slow frequency drifts can be compensated by controlling the crystal temperature with a tuning coefficient of $-3 \mathrm{GHz} / \mathrm{K}$. Before amplification the NPRO beam is passed through an electrooptic modulator (EOM).

Outside the laser box, the amplifier power can be monitored with a photo-detector (PD1) placed behind a highly reflective mirror. The amplifier output beam has a polarization extinction ratio of more than $20 \mathrm{~dB}$. A polarizing beam splitter (Pol-BS1) is used to filter out further possible residual light in unwanted polarization states.

\subsection{Second harmonic generation}

Our experiment uses a detector with a silicon CCD chip whose sensitivity is strongly peaked around the visible spectral region while it approaches zero for wavelengths above $1000 \mathrm{~nm}$ [118]. In the phenomenon of oscillations the regenerated light has the same characteristics as the laser beam in the WISP production vacuum tube [119]. Due to the constrains given by the detector sensitivity we convert the infrared laser light from $1064 \mathrm{~nm}$ to green $532 \mathrm{~nm}$ light exploiting the nonlinear effect of second harmonic generation [120,121] (SHG).

As the nonlinear material we use PPKTP (periodically poled $\mathrm{KTiOPO}_{4}$ ) which shows high conversion efficiencies due to its high intrinsic nonlinearity. It is fabricated from a flux-grown KTP crystal by reorientation of its optical axis in periods of approximately $9 \mu \mathrm{m}$ length by strong electrical poling, leading to a much greater conversion efficiency and an easier phase-matching condition than for conventional SHG crystals [121]. The crystal's dimensions are $1 \mathrm{~mm} \times 2 \mathrm{~mm} \times 2 \mathrm{~cm}$ and its nonlinearity was 
measured to be $d_{\mathrm{eff}} \approx 7.9 \times 10^{-12} \mathrm{~m} / \mathrm{V}$. It is placed inside an oven to stabilize its temperature at around $38{ }^{\circ} \mathrm{C}$ in order to maintain phase-matching of the infrared and green (SHG) waves. Two lenses (L1, L2, see Fig. 3) are used to focus the infrared beam into the crystal to a waist size of $135 \mu \mathrm{m}$. This waist size is a compromise between high conversion efficiency, the risk of damaging the crystal and degradation of the green beam shape. The input polarization can be adjusted to maximum conversion efficiency by a $\lambda / 2$ - plate (P2). The input power level of the infrared beam is set via a variable attenuator consisting of another $\lambda / 2$ - plate (P1) and a polarizing beam splitter (Pol-BS2). Behind the oven the converted green light is separated from the infrared by means of a dichroic mirror (D1) which is followed by a variable attenuator for the green light beam (P3, Pol-BS3) and a collimating lens (L3). Then the light passes a Faraday rotator that forms an optical diode together with the polarizing beamsplitter Pol-BS3 to protect the SHG from any back-reflection from the following optical setup.

The polarization state of the laser light entering the cavity can be adjusted by a combination of a $\lambda / 2$ plate (P4) and a pivotable polarization beam splitter (Pol-BS4). By this combination a clean linear polarization state with an arbitrarily adjustable angle relative to the magnetic field direction can be realized.

With this single-pass SHG scheme a long-term stable output power of $0.8 \mathrm{~W}$ at $532 \mathrm{~nm}$ is available behind D1 when the infrared beam is set to its maximum power level of $35 \mathrm{~W}$.

In steady-state the transversal shape of the green beam acquires a slightly doughnut-like form, probably due to nonlinear absorption processes inside the crystal at high intensities [122]. This slight deviation of the green beam shape from a pure Gaussian lowers the coupling efficiency to the optical resonator to approximately $80 \%$ but does not influence the LSW experiment in any other way.

The relatively small green power poses no problem for the main goal of this paper, namely the successful integration of a large-scale optical resonator into a full and running LSW experiment. It is, however, to be improved when aiming for ultimate sensitivity. To increase the SHG efficiency we plan to change to a resonant SHG by setting up an optical resonator around the nonlinear crystal.

\subsection{Magnet}

The production efficiency of some types of WISPs depends strongly on the strength and length of the background magnetic field (Section 2). The ALPS experiment at DESY uses a spare HERA dipole magnet [123]. More than 400 of these magnets are incorporated in the HERA proton storage ring, which operated from 1991 to end of June 2007.

A HERA dipole magnet has an outer length of $9.8 \mathrm{~m}$ and is operated at cryogenic temperatures of $4.2 \mathrm{~K}$. At ALPS we operate the dipole at a 5.30 T magnetic field. Apart from a few quenches we did not encounter any difficulties.

The bent magnet bore is shielded by an anti-cryostat allowing to perform experiments inside the magnet at room temperature.

Gaseous nitrogen at a constant rate and temperature is flushed through the magnet bore so that it floats around the two vacuum tubes and the end mirror (EM) inserted into the magnet. This stabilizes the temperatures as required for a stable optical path. As mentioned already in Section 3.1 the small clear aperture through the two vacuum tubes imposes special requirements on the laser's beam quality.

The direction of the magnetic field is vertical. Henceforth, the polarization of the laser light inside the cavity will be measured with respect to this direction.

\subsection{Detector and detection bench}

The set-up of the detector bench is sketched in Fig. 3. Reconverted photons from WISPs are reflected by the $45^{\circ}$ main mirror and focused by a lens on a few pixels of the CCD of our camera in order to keep the relevant dark noise as low as possible.

The ALPS experiment uses a commercial astronomy CCD camera SBIG ST-402ME [118] as its detector for regenerated photons. It contains a Kodak KAF-0402ME silicon CCD chip with $765 \times 510$ pixels of $9 \times 9 \mu \mathrm{m}^{2}$. The quantum efficiency is $\approx 60 \%$ for light with a wavelength of $532 \mathrm{~nm}$.

We operate the CCD chip at a temperature of $-5{ }^{\circ} \mathrm{C}$ where it shows a low dark current of 0.03 electrons per pixel per second (corresponding to about 0.05 photons of $532 \mathrm{~nm}$ wavelength)and a readout noise of 17 electrons per pixel, independent of the exposure time. The pixel brightness is measured in ADUs (analogto-digital units) with one ADU corresponding to 1.46 electrons.

The camera allows sampling times between $0.04 \mathrm{~s}$ and $1 \mathrm{~h}$. In order to lower the impact of the readout noise we used $1 \mathrm{~h}$ expositions in our search for WISPs.

In dark frames (exposures with closed shutter) the values of all pixels are found to fluctuate coherently. This is monitored by determining the average pixel ADU for each exposure after cutting against cosmics and other spurious signals. This average fluctuates by up to 30 ADUs and is probably caused by some environmental effects. To compensate for this effect, the ADUs of all pixels in an individual frame are shifted by the difference of the average pixel value in this frame and an arbitrarily defined baseline. After this correction no hint for any additional systematic effect or unknown correlation among the pixels is found.

Special care is required when mounting and fixing all optical components on the detection bench because it has to be removed when the detector tube is pulled out or put into the magnet (as required by the data taking procedure, see Section 5). The exact position of the bench on the supporting structure (fixed to the concrete of the experimental hall) is defined by kinematic seats. In numerous tests it was proved that the focal points of reference beam and alignment beam do not change by more than one pixel on the $\mathrm{CCD}$ after removing and re-installing the detector bench.

In order to monitor pointing stability, a reference beam is split off the main beam in front of the cavity's input coupler (CM) and is guided from the laser hut to the detector bench along a tube located at the side of the magnet. The reference tube is closed from one side with a window to avoid air flows that produce jitter in the reference signal. A small fraction of this reference beam is reflected by the $45^{\circ}$ mirror to the detector box, attenuated and reflected by two mirrors, so that it passes from behind through the main mirror and is focused on the CCD at a location separate from the focus of the main beam. A typical CCD frame with the two regions of interest is shown in Fig. 4.

In future experiments we plan to improve the signal to noise ratio by about a factor of 10 . We will use a new camera with less dark current, less read-out noise and higher quantum efficiency and will improve the focusing of the beam.

\subsection{Vacuum}

To avoid suppression of the photon-WISP oscillation probability both production and regeneration regions are located inside vacuum tubes in which high vacuum conditions are maintained. Taking the oscillation length to be smaller than $8 \mathrm{~m}$ and the photon energy $\omega=2.3 \mathrm{eV}$, the critical mass defined in Section 2 is $M_{\text {crit }} \gtrsim 0.6 \mathrm{meV}$. Assuming that the main component of the residual gas in the tubes is air $\left(\Delta n \simeq 2.9 \times 10^{-4}\right.$ in normal 


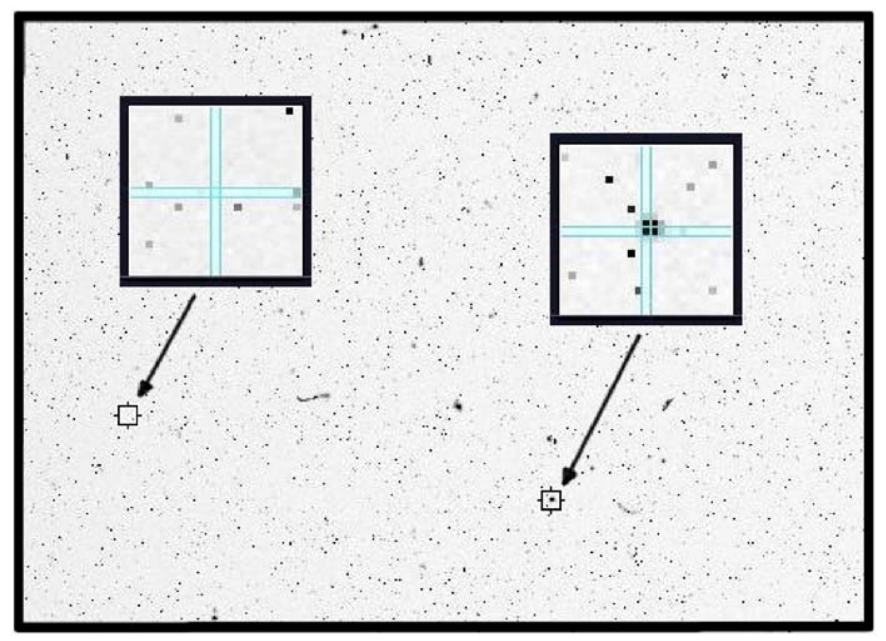

Fig. 4. Selection of a typical $1 \mathrm{~h}$ exposure recorded with the SBIG CCD. Tracks from radioactivity and cosmics are visible as well as warm pixels, i.e. pixels showing large pedestals or high noise rates. The signal (left) and reference (right) regions are shown enlarged. No evidence for photons from WISPs is seen while the reference beam shows up clearly and well focused.

conditions), we can get an upper limit on the pressure,

$P<0.11\left(\frac{T}{273.15 \mathrm{~K}}\right)$ mbar

below which the residual gas will not influence the photon-WISP oscillations. For evacuation of the laser and detector tubes inserted into the magnet we used two HERA turbomolecular pump units. Based on measured outgassing rates, the pressure is estimated nowhere in the vacuum tubes to exceed $10^{-5}$ mbar. So condition (7) is easily satisfied.

\section{The ALPS cavity}

The purpose of optical resonators (cavities) in the context of LSW experiments is to enlarge the light power in the WISP production region relative to the available primary laser power. Such an optical resonator acts therefore as an amplifier for its incident laser power, boosting the sensitivity of the whole experiment. This is possible by coherent superposition of light fields that enter the resonator at different times corresponding to successive roundtrips.

\subsection{Introduction}

The power amplification of a resonator is given by the ratio of the laser power inside the resonator traveling towards the regeneration tube, $P_{\rightarrow}$, to the power incident to the coupling mirror, $P_{\mathrm{CM}}$. This ratio is called power build-up $P B$,

$$
P B=P_{\rightarrow} / P_{\mathrm{CM}} \text {. }
$$

Consider a linear resonator consisting of two mirrors spaced by a distance $L$ fed by a laser of frequency $v$. The first mirror or input coupler $\mathrm{CM}$ has a power transmission coefficient of $T_{\mathrm{CM}}$ and the second one of $T_{\mathrm{EM}}$. In our case, $\mathrm{CM}$ is the mirror situated on the laser table and EM the one placed in the interior the magnet. Absorption, scattering and deflection of the light during one roundtrip are combined into a parasitic loss factor $A$.

During a roundtrip between the mirrors the light acquires a phase $\psi=2 \pi v(2 L) / c$. Resonant enhancement of the circulating light power is achieved when the light wave nearly reproduces itself after one roundtrip, i.e. when $\Phi=\psi \bmod 2 \pi \approx 0$. A cavity has therefore an infinite number of resonances at frequencies $v_{\text {res }, n}=n c /(2 L)$, characterized by integers $n$. The frequency interval between resonances is called free spectral range, $F S R=c /(2 L)$.

Under the assumption that $T_{\mathrm{CM}}, T_{\mathrm{EM}}, A$ and $\Phi$ are all small compared to unity one can approximate the power buildup by [124]

$P B \approx \frac{4 T_{\mathrm{CM}}}{\left(T_{\mathrm{CM}}+T_{\mathrm{EM}}+A\right)^{2}+4 \Phi^{2}}$

which in resonance gives

$P B_{\max } \approx \frac{4 T_{\mathrm{CM}}}{\left(T_{\mathrm{CM}}+T_{\mathrm{EM}}+A\right)^{2}}$.

The errors of these approximations are negligible for all considerations that are made throughout this paper.

The derivative of Eq. (10) with respect to $T_{\mathrm{CM}}$ can be set to zero to find the maximum power build-up with respect to input coupling. This maximum is achieved if the so-called impedance matching condition is fulfilled

$T_{\mathrm{CM}}=T_{\mathrm{EM}}+A$.

Hence, the largest power build-up is obtained when the transmission of the input coupler $T_{\mathrm{CM}}$ is chosen to be as close as possible to the sum of parasitic losses $A$ and output coupling of the cavity $T_{\mathrm{EM}}$.

Derivation of Eq. (10) with respect to $A$ or $T_{\mathrm{EM}}$ shows that there is no maximum of $P B_{\max }$ over these parameters clarifying that it is always best to keep parasitic losses and the output coupling as small as possible in order to maximize the power build-up. But, even when choosing the best optics available and assembling the cavity under as clean ambient conditions as possible, some parasitic losses will always remain. Hence, one normally has to guess $A$ in advance during the design process of an optical resonator. The transmission coefficient of the input coupler can then be chosen to maximize $P B_{\max }$.

To realize the full possible power build-up in the cavity, the alignment, shape and resonance frequency of the resonator eigenmode must be exactly matched by the incident laser beam. These parameters are defined by the alignment and radii of curvature $(R O C)$ of the cavity mirrors and by the optical distance between them [125].

Single mode continuous-wave lasers emit most of their power into the fundamental transversal mode $\mathrm{TEM}_{00}$. It is therefore most efficient to use spherical mirrors for the resonator such that the laser mode can be easily matched to the eigenmode of the cavity. However, every slight mismatch in beam shape or alignment causes some fraction of the incident power to overlap with higher order transversal eigenmodes of the cavity. In general, the higher order spatial modes have different resonance frequencies than the fundamental mode. Hence, this power fraction is not coherently enhanced when the fundamental mode is resonant. This effect is called mis-modematching and it has to be minimized in order to maximize the intra-cavity power. In turn, such a cavity with nondegenerated resonant frequencies has the positive effect of behaving like a mode filter for the production beam, which also restricts the beam of re-converted photons to exist only in the $\mathrm{TEM}_{00}$ mode. Such a beam can then be focused to smaller spot sizes than beams consisting of multiple higher order transversal modes, which is a key issue when maximizing the signal to background ratio in our CCD camera. The full theory on mode structures of laser beams and resonators can be found in several publications, for instance Refs. [124-126].

Recall that, in addition to an optimized spatial overlap, the laser frequency has to match one resonance frequency $v_{\text {res. }}$. Small fluctuations of the cavity length $\Delta L$ induce correspondingly small relative changes of its resonance frequency given by 
$\Delta v_{\text {res }} / v_{\text {res }}=-\Delta L / L$. To show how even tiny length changes degrade the power build-up, we solve Eq. (9) for an absolute length change $d$ that would cause a reduction to one half of its resonant value:

$d= \pm \frac{1}{8 \pi} \lambda\left(T_{\mathrm{CM}}+T_{\mathrm{EM}}+A\right)$ for $\frac{P B}{P B_{\max }}=\frac{1}{2}$.

From this expression (and bearing in mind that we are interested in the smallest possible values of $T_{\mathrm{CM}}+T_{\mathrm{EM}}+A$ to maximize the power build-up) it becomes clear that even length fluctuations on scales much smaller than the laser wavelength will cause huge changes of the power build-up of the cavity.

A similar constraint for the necessary stability of the laser frequency can be deduced from Eq. (12) if one substitutes $\lambda=c / v$ and solves for the laser frequency $v$ at which the power build-up is reduced to half its peak value. The distance between these two points on the frequency axis is called the full width at half maximum (FWHM) linewidth of the optical resonator and is given by

$\Delta v=F S R \frac{\left(T_{\mathrm{CM}}+T_{\mathrm{EM}}+A\right)}{2 \pi}$.

The laser frequency must be kept well within this FWHM linewidth to keep the power build-up near its peak value. The ratio FSR/FWHM, which is a measure for the cavity's frequency selectivity, is usually called finesse.

In order to keep the power build-up stable, the difference between the resonance frequency and the laser frequency can be minimized by a feed-back control system. This can either actuate on the cavity length or on the laser frequency.

Once spatial modes and frequencies of the laser and the cavity are matched, the cavity will exhibit its full power build-up. This gain factor will remain fairly constant while scaling the incident power until it gets spoiled by thermal distortion or even by the destruction of the optics.

The power inside the cavity can be easily measured by monitoring the power transmitted through the end mirror, $P_{\mathrm{EM}}$ since, under the outlined approximations

$P_{\mathrm{EM}}=T_{\mathrm{EM}} P_{\rightarrow}$.

\subsection{Design of optical resonator}

The design of the ALPS optical resonator was constrained by several aspects. First, the site at DESY does not allow for the use of two magnets in a row so the production and regeneration regions of our LSW experiment must be located inside the same magnet. This requires that one mirror of the cavity has to be placed in the middle of the HERA magnet. Second, the bent magnet bore has an cat's pupil-shaped clear inner aperture of only $28 \mathrm{~mm}$ height and $14 \mathrm{~mm}$ width (see Ref. [57]).

A linear resonator comprised by two mirrors was realized. One mirror, $\mathrm{CM}$, is held in place by a rigid and manually adjustable mirror mount on an optical table in front of the magnet and the other mirror, EM, is mounted near the middle of the magnet on a self-made small and non-magnetic mirror holder attached to the end of the WISP production vacuum tube [58], see Fig. 5. The mirror holder for EM was designed such that it allows for remote alignment to compensate drifts. It can be tilted around two perpendicular directions using two high magnetic field compatible Picomotors $^{\mathrm{TM}}$ custom made by New Focus ${ }^{\mathrm{TM}}$. The distance between both mirrors is $8.6 \mathrm{~m}$ most of which is occupied by a vacuum tube with two anti-reflectively coated windows (AR windows).

Several stable resonator designs that differ in the radii of curvature of the cavity mirrors are possible [124,125]. Our

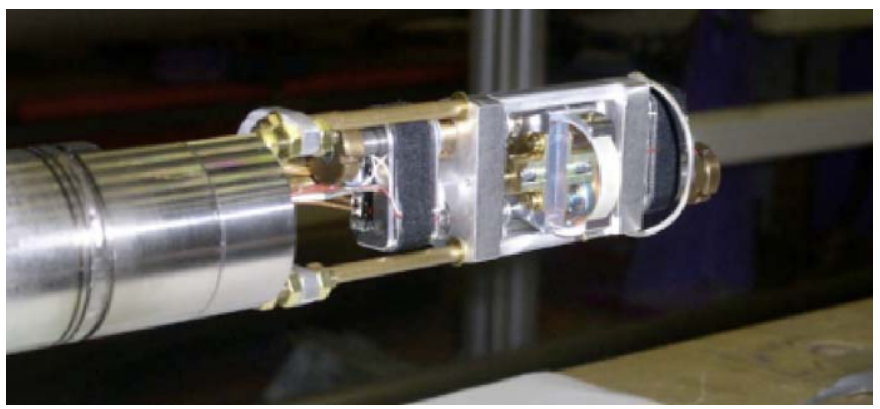

Fig. 5. Detail of the non-magnetic holder of the end mirror EM.

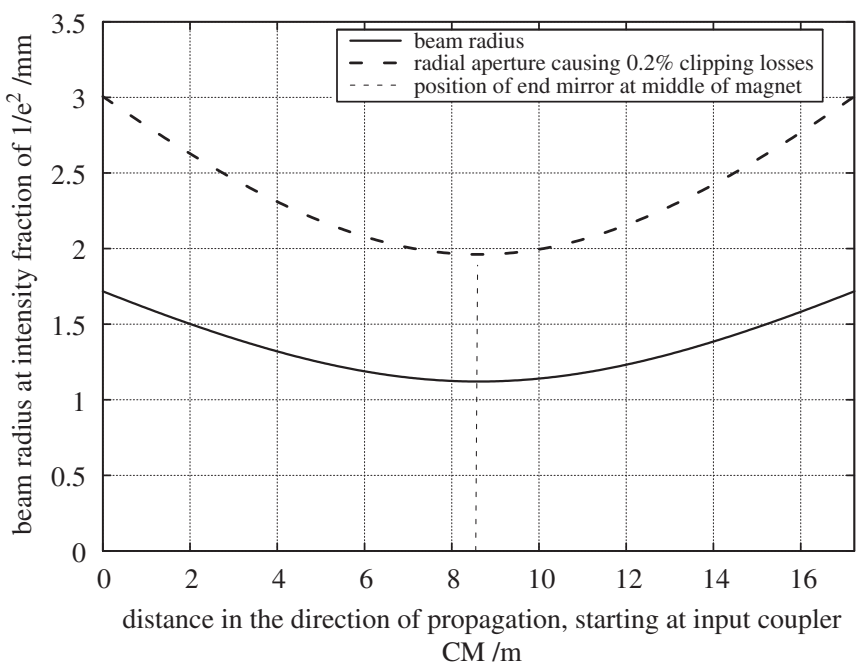

Fig. 6. Beam radius of the fundamental eigenmode of the ALPS optical resonator together with the radius at which $0.2 \%$ of the mode's power would be clipped. The position of the end mirror EM is shown as a vertical line. Clearly, the beam size is always well below the minimum aperture of our production vacuum tube (14 mm).

resonator uses a plano-concave design with one plane mirror (EM) and a curved one with $R O C=-15 \mathrm{~m}$. The resulting fundamental cavity eigenmode needs a free circular aperture of only $6 \mathrm{~mm}$ diameter to keep power losses per roundtrip due to clipping below $0.2 \%$. The resonance frequencies of all higher order transversal eigenmodes up to a mode index sum of 10 do not match the $\mathrm{TEM}_{00}$ resonance frequency within a linewidth. This simplifies the readout of the length changes of the cavity, which is necessary for the feed-back control system explained below. The evolution of the beam radius of the chosen fundamental eigenmode is shown in Fig. 6 together with the required free circular aperture to keep losses due to clipping below the value mentioned above. Here "beam radius" corresponds to the lateral distance from the beam's propagation axis where the Gaussian intensity distribution of the fundamental $\mathrm{TEM}_{00}$ has fallen to a fraction of $1 / e^{2}$.

As end mirror (EM) we chose a highly reflecting mirror whose measured transmission is $T_{\mathrm{EM}}=170 \mathrm{ppm}$. Because our resonator includes four antireflective coated surfaces of the vacuum tube's windows (see Fig. 3), with rest reflection coefficient of $R \lesssim 0.3 \%$ each, we chose a power transmission design value of approximately $2 \%$ for the input coupler. Accurate measurements on CM resulted in a power transmission of $T_{\mathrm{CM}} \approx 2.3 \%$. Assuming the realization of an impedance matched cavity according to Eqs. (10) and (13) these values would result in a maximum power build-up of $P B_{\max } \approx 43$ and a FWHM of $\Delta v \approx 130 \mathrm{kHz}$. 
In order to get as close as possible to this theoretical value, an optimization of the spatial overlap of laser and resonator mode has to be performed. This can be done with the two beam shaping lenses L4 and L5, the two alignment mirrors AM1 and AM2 and with the two adjustable mounts of the cavity mirrors CM and EM (see Fig. 3). If the incident laser beam as well as the cavity mode are perfect Gaussian $\mathrm{TEM}_{00}$ modes, then the combination of these actuators must be sufficient to maximize the spatial overlap in all its degrees of freedom.

\subsection{Design of stabilization feedback control}

The experimental site at DESY is exposed to strong acoustic and vibrational influences. These ambient conditions give rise to large and fast length fluctuations of our cavity as well as significant alignment fluctuations with strong impact on the power build-up. In order to compensate these fluctuations we constructed an electronic feedback control loop that actuates on the laser frequency.

The difference between the laser frequency and the actual resonance frequency of the cavity is determined via a sideband modulation spectroscopy technique called Pound-Drever-Hall (PDH) readout scheme, with the help of a photodiode PD3 whose output is high-pass filtered [127-129]. The necessary phase modulation sidebands are generated by an electro-optic modulator (EOM) which is part of the laser system (see Section 3.1). After demodulation the resulting error signal is filtered by a controller and then amplified by a fast high voltage amplifier. The resulting high voltage signal is then passed to the fast frequency actuator (a piezoelectric transducer acting on the NPRO crystal of the laser, not shown in Fig. 3 for simplicity) which is part of the laser system (see Section 3.1). This control loop has a bandwidth of approximately $28 \mathrm{kHz}$ limited by mechanical resonances of the frequency actuator around $200 \mathrm{kHz}$.

The length fluctuations of the cavity are much larger than the range over which the PDH scheme would provide a valid error signal. Therefore, if the control loop is closed at an arbitrary cavity state the controller would in most cases not be able to bring cavity and laser into resonance. To solve this problem the loop is equipped with automatic lock acquisition electronics that monitors the light power that is reflected back from the cavity via the DC signal of PD3. If the locked state is lost, the reflected power rises above a user-defined level which is the trigger to scan the laser frequency and search for the next resonance. If this is found the system is automatically relocked. Intervention by an operator is not necessary.

We record the DC signal of PD3 every $30 \mathrm{~s}$ in order to keep the information about the intracavity power for the data analysis. This signal was calibrated in test runs by measuring simultaneously the light passing through the mirror EM with known transmission $T_{\mathrm{EM}} \approx 170 \mathrm{ppm}$.

\subsection{Characterization of the production resonator}

After installation the cavity was first characterized at low incident powers $P_{\mathrm{CM}}$ around $50 \mathrm{~mW}$. The stabilized fundamental eigenmode of the ALPS cavity is observed with a CCD camera behind the mirror EM. It has a nice round shape without any observable fluctuations or distortions. This is important for the detection stage of our LSW experiment because the position and intensity distribution of a possible beam of re-converted photons behind the wall is then stationary and well defined by the orientation and profile of the cavity mode (see Section 3.4). Furthermore, this is a first hint suggesting that the cavity mode does not suffer from large losses due to extensive clipping or heavy absorption.

According to Eq. (10) optical losses inside the cavity would reduce the achievable power build-up. Due to its length of $8.6 \mathrm{~m}$ and the way it must be mounted inside the magnet, it is not possible to assemble the cavity in a cleanroom environment. Therefore, it is important to check if additional internal losses might be caused by dust or other impurities.

In Section 4.2 we expected $P B_{\max } \approx 43$ from our cavity design. We measured this quantity by recording the power transmitted through EM and determining the intra-cavity power $P_{\rightarrow}$ through Eq. (14) in a dedicated setup. We achieve a power build-up of $P B=44 \pm 2$ which at first glance seems to agree nicely with our expectations.

However, a fast scan of the laser frequency over more than one free spectral range reveals that $\approx 20 \%$ of the incident power $P_{\mathrm{CM}}$ is coupled to different higher order transversal modes. This value could not be reduced even by careful optimization of the spatial overlap between laser beam and cavity mode. Therefore, it most likely originates from distortions of the incident beam shape to some non-Gaussian intensity profile by the SHG and from alignment fluctuations of the long cavity [126]. In order to compare our measurement of $P B$ with the value of $P B_{\max }$ obtained in the previous section one has to correct for the amount of $P_{\mathrm{CM}}$ in higher order transversal modes. This correction for the mismodematching results in a measured power build-up in the $\mathrm{TEM}_{00}$ mode of

$P B_{\mathrm{TEM}_{00}}=55 \pm 3$

which is slightly higher than expected.

Additionally, a value of $\Delta v \approx 130 \mathrm{kHz}$ was expected. A direct measurement of this value resulted in $\Delta v=127 \pm 12 \mathrm{kHz}$, in excellent agreement with the expectations.

Therefore, we have two independent ways of determining the internal losses in the cavity. Using the measured values of $P B_{\mathrm{TEM}_{00}}$ and $\Delta v$ with Eqs. (10) and (13) we obtain for the parasitic losses introduced by the antireflective coated surfaces inside our cavity values of $0.22 \pm 0.01 \%$ and $0.28 \pm 0.05 \%$ per facet, respectively. These values are compatible with each other. They turn out to be slightly smaller than the estimation used for the design of the resonator, which explains the fact that $P B_{\mathrm{TEM}_{00}}$ surpasses our expectations. Clearly, the windows inside the cavity dominate the overall losses. Eliminating the windows in future experiments could be the first step towards a significantly enlarged power build-up.

To characterize the adequacy and performance of our frequency stabilization control loop we further investigate the free-running and stabilized rms fluctuations of the frequency mismatch between laser and cavity. The measured spectral densities are shown in Fig. 7. Because the power build-up is seriously degraded if the frequency mismatch amounts to more than half of the cavities FWHM all measurements in Fig. 7 are normalized to its measured value of $\Delta v=127 \mathrm{kHz}$. The figure shows the squared sum of the measured noise spectral densities of the control signal applied to the frequency actuator of the laser and of the error signal of the control loop. This gives an estimate on the size of the mismatch in an uncontrolled situation (freerunning). Additionally, the measured noise spectral density of the error signal of the control loop itself is shown which reflects the remaining mismatch once the control loop is closed. Furthermore, a line is plotted that gives a rough estimate of the free-running laser frequency noise.

Because the linear spectral density of the overall frequency mismatch (Fig. 7, d) is given by the squared sum of cavity length noise and laser frequency noise (c), one can deduce from the large discrepancy between (d) and (c) that the by far greatest 


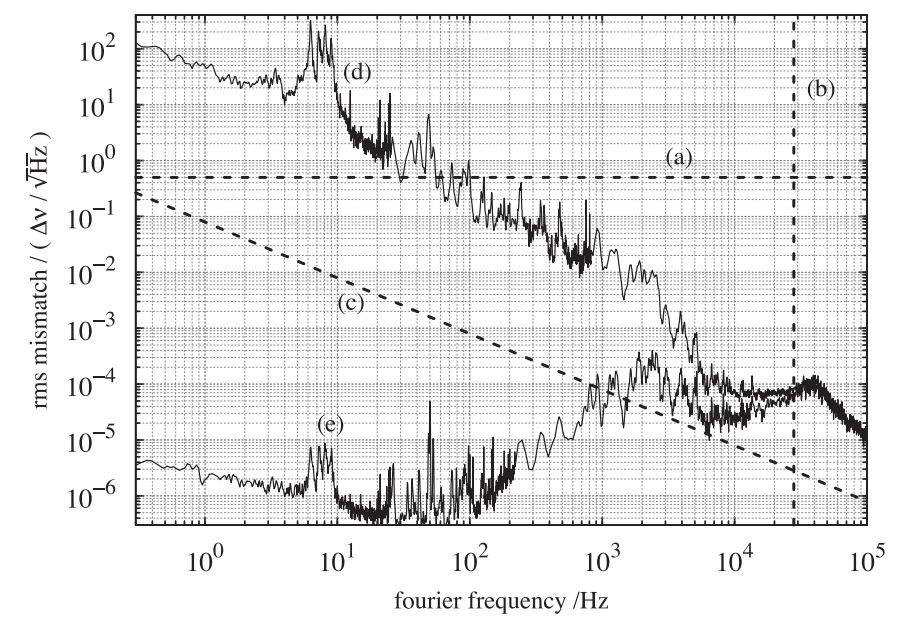

Fig. 7. Comparison of rough upper limit fit to free-running laser frequency noise (c), that was taken from Ref. [130], with measurements of the free-running (d) and stabilized (e) mismatch between the laser and cavity resonance. The free-running mismatch is effectively suppressed for Fourier frequencies smaller than the bandwidth of the control loop (b). The power build-up is seriously degraded for frequencies with larger rms noise than one half of the cavities FWHM linewidth $\Delta v$ (a).
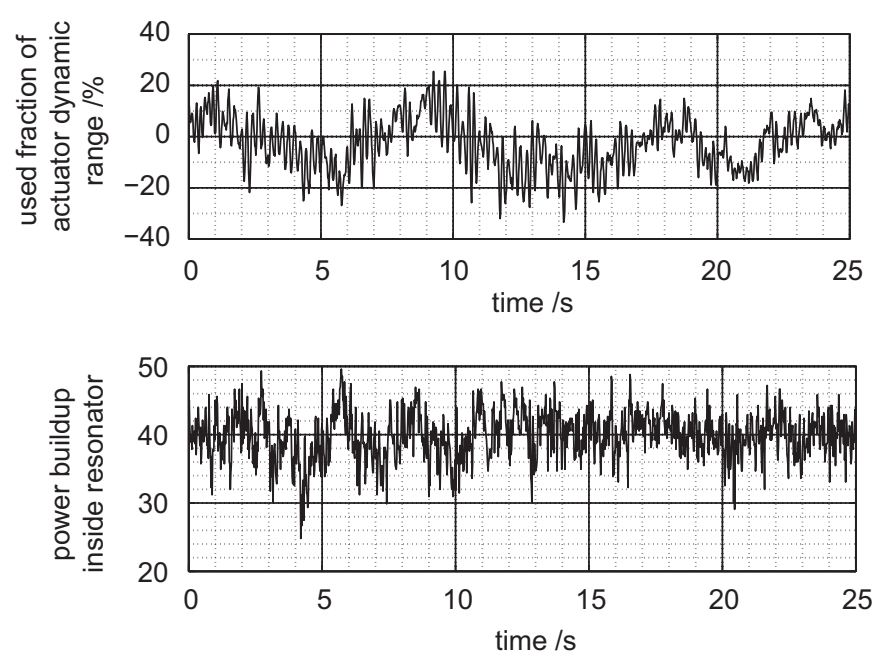

Fig. 8. Typical time series of the power build-up (bottom panel) and the actuator signal (top panel) while the electronic frequency stabilization control loop is active.

contribution to the overall free-running frequency mismatch is due to cavity length noise. For Fourier frequencies below $100 \mathrm{~Hz}$ this free-running rms length noise causes resonance frequency fluctuations much greater than one half of the cavities FWHM linewidth reducing the average power build-up close to zero. But when the active stabilization system is turned on the rms frequency mismatch is largely reduced. Their remaining impact on the power build-up is virtually negligible.

Our control loop is capable of stabilizing the laser to the cavity on longer timescales as well. In Fig. 8 a typical time series of remaining power build-up fluctuations under lock is displayed. One can see that the laser light is kept resonant inside the cavity for tens of seconds. The dynamic range of $<40 \%$ of the frequency control actuator is needed during this time so that slow drifts over even longer timescales can still be compensated.

The remaining power build-up fluctuations probably originate from changes of the alignment of the incident laser beam relative to the eigenmode of the resonator. An additional auto-alignment system may improve this situation and increase the average power build-up slightly.
As far as the frequency control loop is concerned, very slow drifts of the cavity length saturate the actuator on timescales of some tens of minutes causing the stabilization to fail and hence the power build-up to be lost. In that case a fast relock was realized by means of the already mentioned automatic lock acquisition electronics and hence long measurement periods were possible.

A timeseries of the power build-up during such a relock sequence is shown in Fig. 9. To stimulate a loss of the locked state of the resonator strong mechanical length fluctuations were introduced into the system by deliberately hitting the optical table. While this disturbance is ringing down the stabilization fails several times because of limited dynamic range which causes the power build-up to drop to zero. This is detected by the automatic lock acquisition electronics that reacquire the locked state every time within only fractions of a second.

In order to characterize the impact of alignment fluctuations and to correlate the internal cavity power with the power reflected by CM we performed a set of calibration measurements. Their results are used to monitor the power in the cavity during data taking. For the calibration we introduced manually different

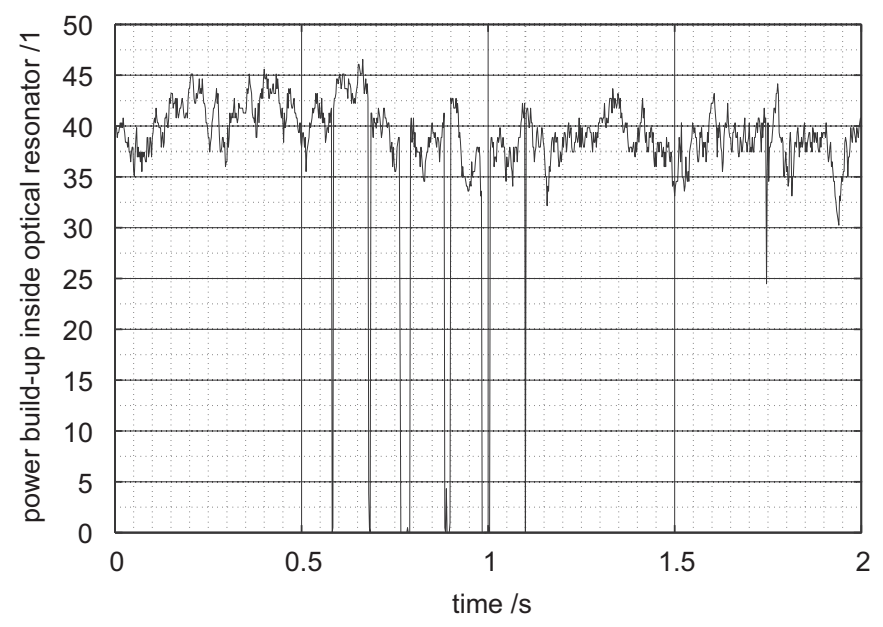

Fig. 9. Typical power build-up inside the optical resonator during multiple automatic relock sequences caused by deliberately introducing a strong vibrational disturbance of the cavity length.

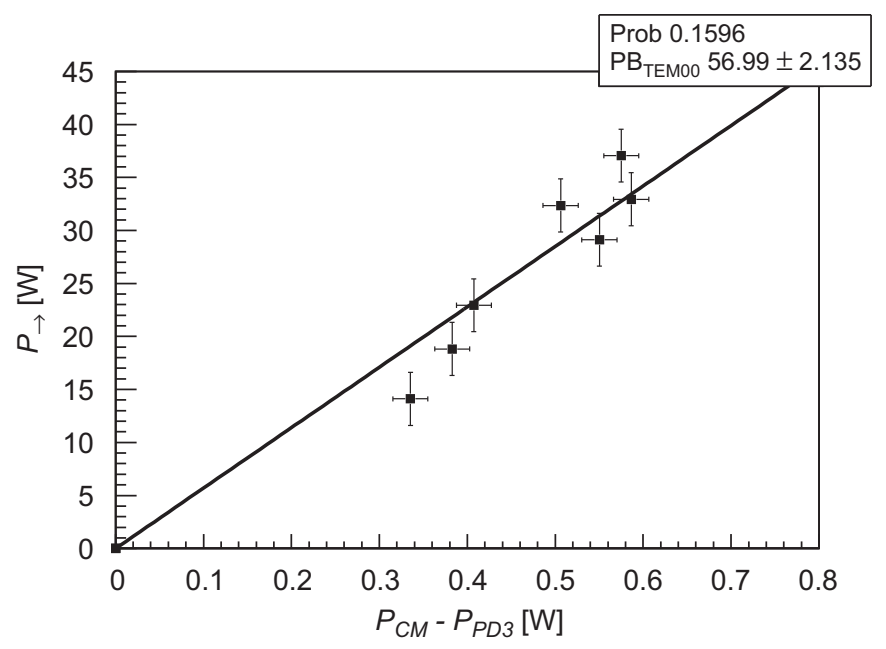

Fig. 10. Power inside of the cavity, $P_{\rightarrow}=P_{\mathrm{EM}} / T_{\mathrm{EM}}$, as function of the power fed into the cavity, $P_{\mathrm{CM}}-P_{\mathrm{PD} 3}$ where $P_{\mathrm{CM}}$ was kept equal to $0.8 \mathrm{~W}$ during the different measurements and $P_{\mathrm{PD} 3}$ denotes the cavity's reflected power as measured by PD3. The slope gives by definition $P B_{\mathrm{TEM}_{00}}$. 
small misalignments of the laser direction and the cavity's eigenmode and measured the power leaking through the mirror EM and the PD3 signal while the cavity was locked. The results are shown in Fig. 10. They show how the intra-cavity power $P_{\rightarrow}$ is degraded by small misalignments and how this is tracked by the power reflected by $\mathrm{CM}$. The slope of this relation gives by definition the power build-up $P B_{\mathrm{TEM}_{00}}=57 \pm 2$. This is in agreement with the value of $55 \pm 3$ given in Eq. (15) thus demonstrating the overall consistency of our understanding of the cavity.

Finally, let us turn to the characterization of the polarization. The laser light enters the optical cavity with a well defined linear polarization. However, we have generally observed that this state changes inside the cavity. This is correlated with the fact that the AR windows are not perfectly perpendicular to the beam. We confirmed this by deliberately changing the angle between windows and laser light and observing the corresponding change of the polarization. The elimination of the AR windows inside the cavity should therefore solve this problem.

\section{Data taking and analysis}

The data taking at the ALPS experiment proceeds in three steps. First, the regeneration tube is installed without the wall. The small fraction of laser light transmitted through the end mirror (EM) attached to the end of the production tube is used to align the detector bench. In the second step, the detector tube is removed, the wall is attached, the detector tube is reinstalled and evacuated and data taking takes place. After a certain period (typically one week) the detector tube is removed again, the wall detached and the open tube re-installed to check the alignment. This third step marks the end of one data taking period.

While data taking, $1 \mathrm{~h}$ exposures are recorded by the CCD. Only frames for times where no technical problems (i.e. magnet quenches) occurred are kept for further analyses. These frames are checked for cosmics and other spurious tracks from radioactivity in a region of $25 \times 25$ pixels around the expected position of re-converted photons from WISPs. About $10 \%$ of all $1 \mathrm{~h}$ frames are rejected by this requirement.

Finally, re-converted photons are searched for by comparing the averaged sum of the ADU counts of all pixels in the signal region (see below) in data frames and dark frames. The latter ones are recorded with closed camera shutter and typically also with the magnet switched off and no or very low intensity laser light. Hence, any excess in the data frames could be a signature for WISP production.

It should be stressed that this simple approach holds true as long as no hint for an excess is derived from the data. If an excess is found, more detailed studies are to be performed demonstrating the WISP origin of the recorded photons.

\subsection{Signal search}

As sketched above, we used light-shining-through-the-mirror to align the detector optics and to define the search region for reconverted photons on the CCD. However, a systematic difference between the impact position of the light used for alignment and the light from photon-WISP-photon conversions arises from the non-perfect parallelism of front and back surface of the cavity end-mirror (EM). The traversing light is bent by the "wedge" shape of the mirror while the WISPs pass the mirror unaffected. We used two methods to determine the angle of the wedge:

- The mirror is rotated and the diameter of the circular path of a distant beamspot is measured.
- The angular distance between the light passing through the mirror and the secondary reflection from the uncoated backside of the mirror is measured.

Both methods give an angle of $0.017^{\circ}\left(0^{\circ}\right.$ for parallel front and back surfaces). With a distance of $\approx 8 \mathrm{~m}$ between mirror and CCD and a lens in front of the CCD with a focal length of $60 \mathrm{~mm}$ one arrives at a difference of $\approx 8 \mu \mathrm{m}$ between the position of the beamspots from the light used for alignment and the location of the re-converted photons from WISPs. The CCD has a pixel size of $9 \mu \mathrm{m}$, hence the search region is to be shifted by one pixel relative to the beamspot used for alignment (in our case upwards). The analogous effects caused by the AR windows close to the EM (inside the cavity) and at the end of the detector vacuum tube were also tested. However, the resulting angles were found to be negligible.

The beamspot profile of the re-converted photons is exactly the same as the beamspot used for alignment because the reconverted photons will have the same properties as the laser photons. The secondary reflection mentioned above will hit the CCD 5 pixels below the primary beamspot and is, as a conservative approach, not subtracted here because of its low intensity $(<4 \%$ of the primary spot). Therefore, the signal profile used in this analysis is a little wider than the beamspot profile of re-converted photons from WISPs. Fig. 11 shows the measured beamspot profile.

Taking this beamspot profile and assuming a possible misalignment of \pm 1 pixel in each direction (corresponding to the repositioning accuracy mentioned above) in the worst case (shift +1 pixel in $x$ and -1 pixel in $y$ ) $58 \%$ of the signal is contained in a $5 \times 5$ pixel large search region. Without a shift this value is above 95\%. Therefore, we assume in the following that on average $(75 \pm 15) \%$ of the signal is contained in the search region.

A hypothetical signal is searched for by comparing the mean value of the sum of the ADUs in the 25 pixels of the signal region in data frames and dark frames after correcting each frame for the pedestal fluctuations.

One principle difference in both frame sets is the presence of light from the reference beam in the data frames. The photon flux of the reference beam at the CCD is about $6.5 \mathrm{~Hz}\left(2.4 \times 10^{-18} \mathrm{~W}\right)$. By comparing dark frames and frames with the reference beam shining on the CCD we proved that the reference light does not affect the signal region. The distance between the signal region and the reference beam spot position amounts to $2 \mathrm{~mm}$ on the chip (230 pixels, see Fig. 4).

After performing the pedestal correction, the dark frame data sets taken over the course of two years agree within statistical uncertainties. No long term drift or other changes are observed.

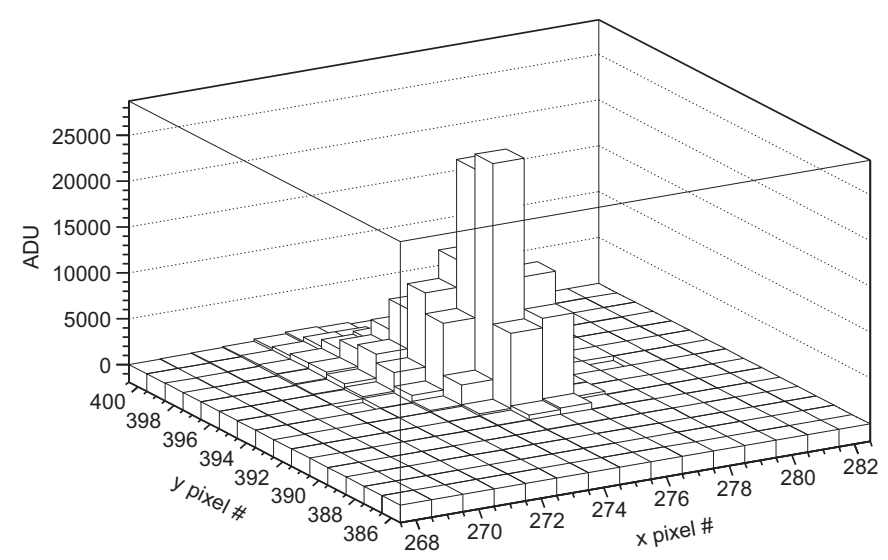

Fig. 11. The beamspot profile of the laser light leaking through the mirror in the centre of the ALPS magnet. 
As a final check the dark frame data set (recorded in the course of 24 days) used for the analysis shown below is divided into a first and second half (104 frames each). Mean and width of the distribution of the sum of the pixels in the signal region agree very well within statistics, so that no hint on systematic differences is found.

\subsection{ALPS exemplary run}

The data set used for this analysis consists of 25 CCD frames with an exposure of $1 \mathrm{~h}$ each. These frames were selected out of 31 frames taken during one week. Three frames, where a magnet quench occurred, are not taken into account for the data analysis. Another three frames are rejected, because the visual inspection of these frames shows hints for cosmic ray activity or radioactivity in the $25 \times 25$ pixel region around the position for the expected signal.

One hour long dark frames with the camera shutter closed were recorded to be compared with the data frames. After cutting against cosmics and radioactivity as described above 208 frames remain for the analysis. The number of dark frames is a factor of 8 larger than the data frames thereby guaranteeing that the statistical accuracy of our flux measurement is dominated by the number of data frames.

The cavity internal power during data taking is shown in Fig. 12. We achieved

$P_{\rightarrow}=34 \pm 4 \mathrm{~W}$

for the production of WISPs. The uncertainty originates from a conservative estimate of the systematic uncertainty of the calibration procedure, see Fig. 10.

The linear polarization state of the eigenmode of the cavity during the data taking was measured (behind the mirror EM) to be $55^{\circ}$ with respect to the orientation of the magnetic field. We have thus $67 \pm 10 \%$ of the laser photons with perpendicular polarization and $33 \pm 10 \%$ with parallel polarization, see Table 2 .

From the alignment test before data taking the central position of the search region was determined to be at the $(276,391)$ pixel coordinates of the $\mathrm{CCD}$, while the alignment test after data taking gave the position $(275,392)$. The difference by \pm 1 pixels matches our experience for the re-positioning accuracy derived from numerous tests before. The distribution of the ADU sums in the search region is presented in Fig. 13 for both data and dark frame

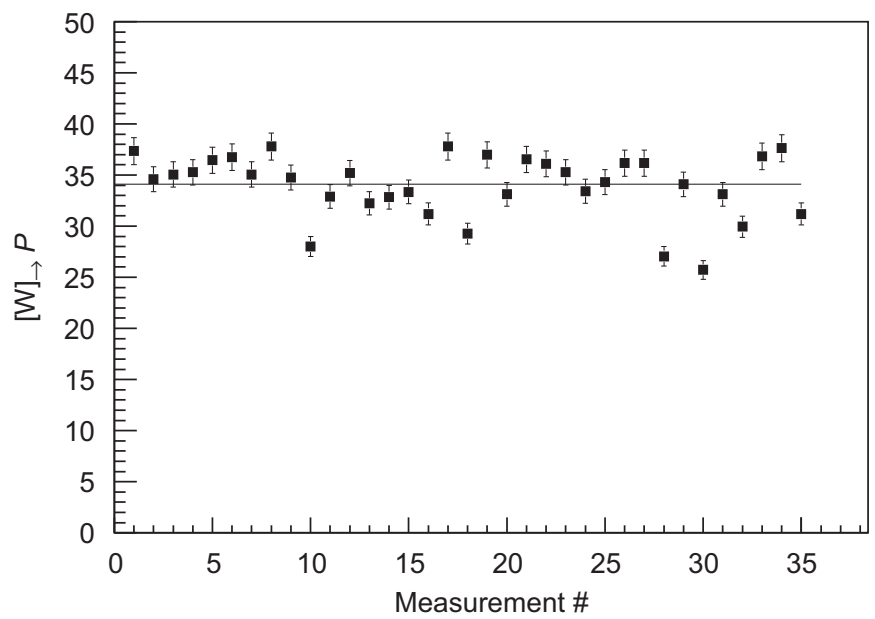

Fig. 12. Monitored power in the optical resonator during the collection of the data frames. The laser power was recorded each $30 \mathrm{~s}$; only each hundredth point is shown for simplicity. The average power relevant for the measurements $\left(P_{\rightarrow}\right)$ is determined to be $34+4 \mathrm{~W}$. This corresponds to a power build-up of $43 \pm 5$ in excellent agreement with the characterization described in Section 4.4.
Table 2

Effective laser power relevant to the search for WISPs.

\begin{tabular}{ll}
\hline Polarization & Flux $(\mathrm{Hz})$ \\
\hline Parallel & $(3.0 \pm 0.9) \times 10^{19}$ \\
Perpendicular & $(6.1 \pm 0.9) \times 10^{19}$ \\
Independent & $(9.1 \pm 0.1) \times 10^{19}$ \\
\hline
\end{tabular}

Parallel, perpendicular and independent denote the orientation of the laser light polarization with respect to the magnetic field direction.
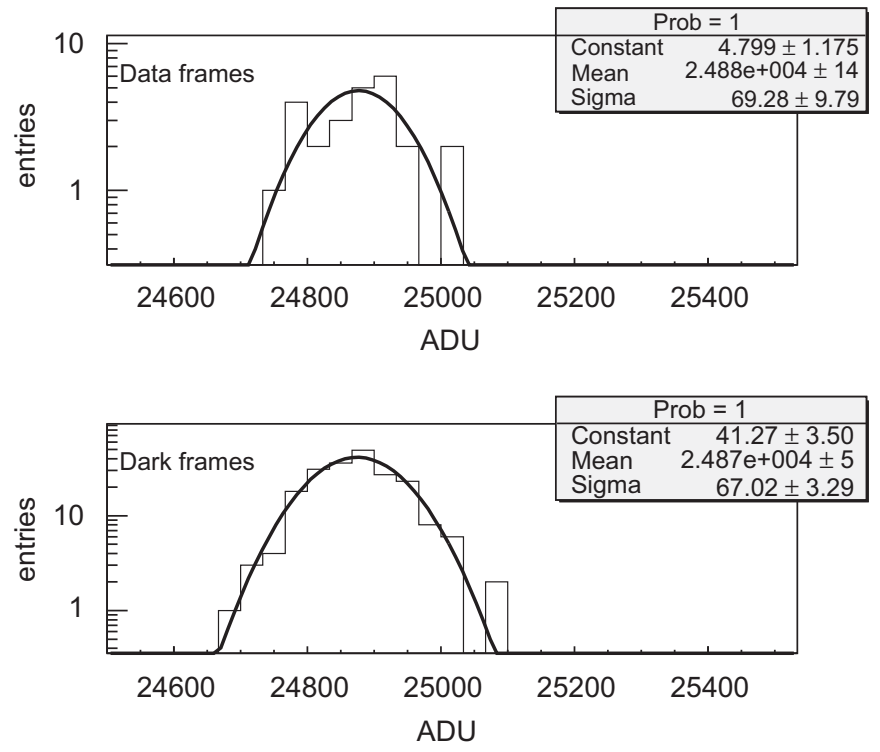

Fig. 13. Distribution of the sums of the ADUs in the $5 \times 5$ pixels defining the search region for photons from re-converted WISPs. Each CCD frame ( 25 data frames, top, and 208 dark frames, bottom) gives one entry. The boxes show the results of Gaussians fitted to the distributions. Due to the dark current and pedestal values each pixel shows an ADU of about 995 on average.

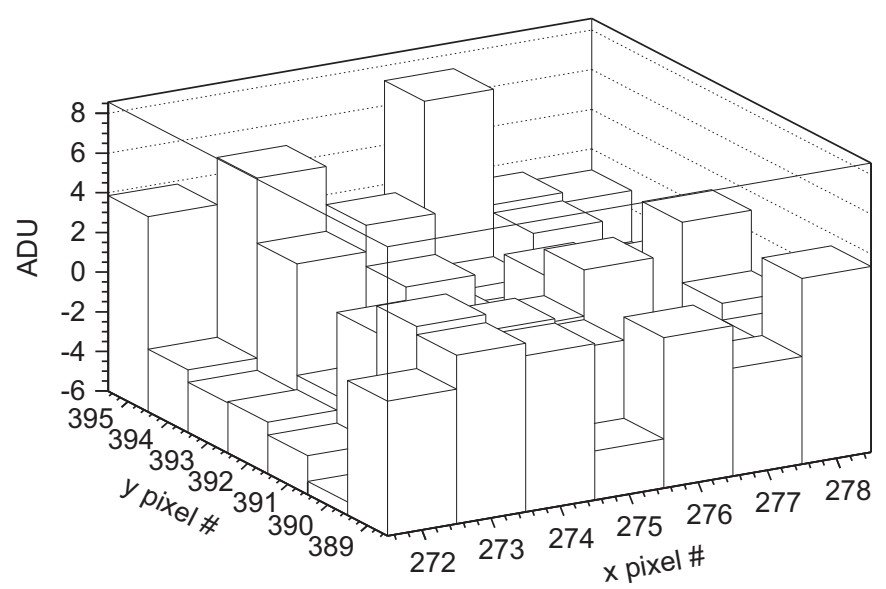

Fig. 14. The difference between the signal (average of 25 data frames) and background (average of 208 dark frames) ADUs shows no excess above noise expectations. A signal of photons from re-converted WISPs should be centered around the position $(275 \pm 1.392 \pm 1)$.

Table 3

The difference of the average of the sums of ADU values of data and dark frames in the search region is shown in the first column.

\begin{tabular}{llll}
\hline ADU diff. & Photon \# & Flux & Limit [95\% C.L.] \\
\hline $2.6 \pm 14.6$ & $8 \pm 49$ & $(2 \pm 13) \mathrm{mHz}$ & $29 \mathrm{mHz}$ \\
\hline
\end{tabular}

No significant excess is observed. The result is used to determine a 95\% C.L. flux limit for photons from re-converted WISPs. 
Table 4

Probability of "light shining through a wall" tested with the ALPS exemplary run and corresponding upper limits.

\begin{tabular}{lllc}
\hline Polarization & Prob. $\times 10^{22}$ & $95 \%$ C.L. & 99\% C.L. \\
\hline Parallel & $0.8 \pm 4.4$ & 9.4 & 12 \\
Perpendicular & $0.4 \pm 2.2$ & 4.5 & 5.8 \\
Independent & $0.3 \pm 1.4$ & 3.0 & 3.9 \\
\hline
\end{tabular}

Parallel, perpendicular and independent denote the orientation of the laser light polarization with respect to the magnetic field direction. sets. Each frame is corrected for the pedestal fluctuation (see above) and gives one entry.

It is obvious that there is no hint for any signal in the data frames: mean and width of both distributions agree very well within statistics. Also searching for a signal at other positions (see Fig. 14) does not reveal any significant excess. Hence we do not observe any evidence for WISP production. The nice agreement of data frames and dark frames also confirms that no light of the reference beam is scattered into the signal region.

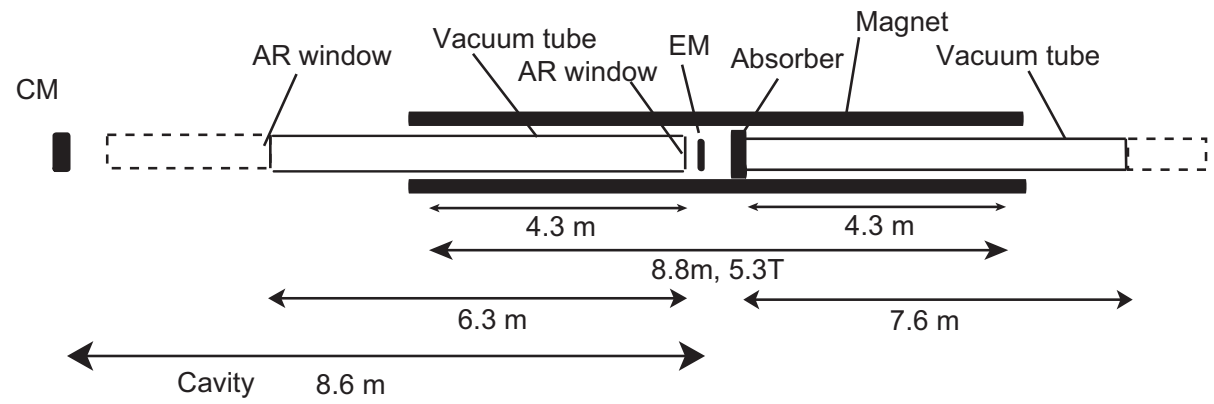

Fig. 15. Summary of the relevant experimental parameters for the ALPS WISP search.
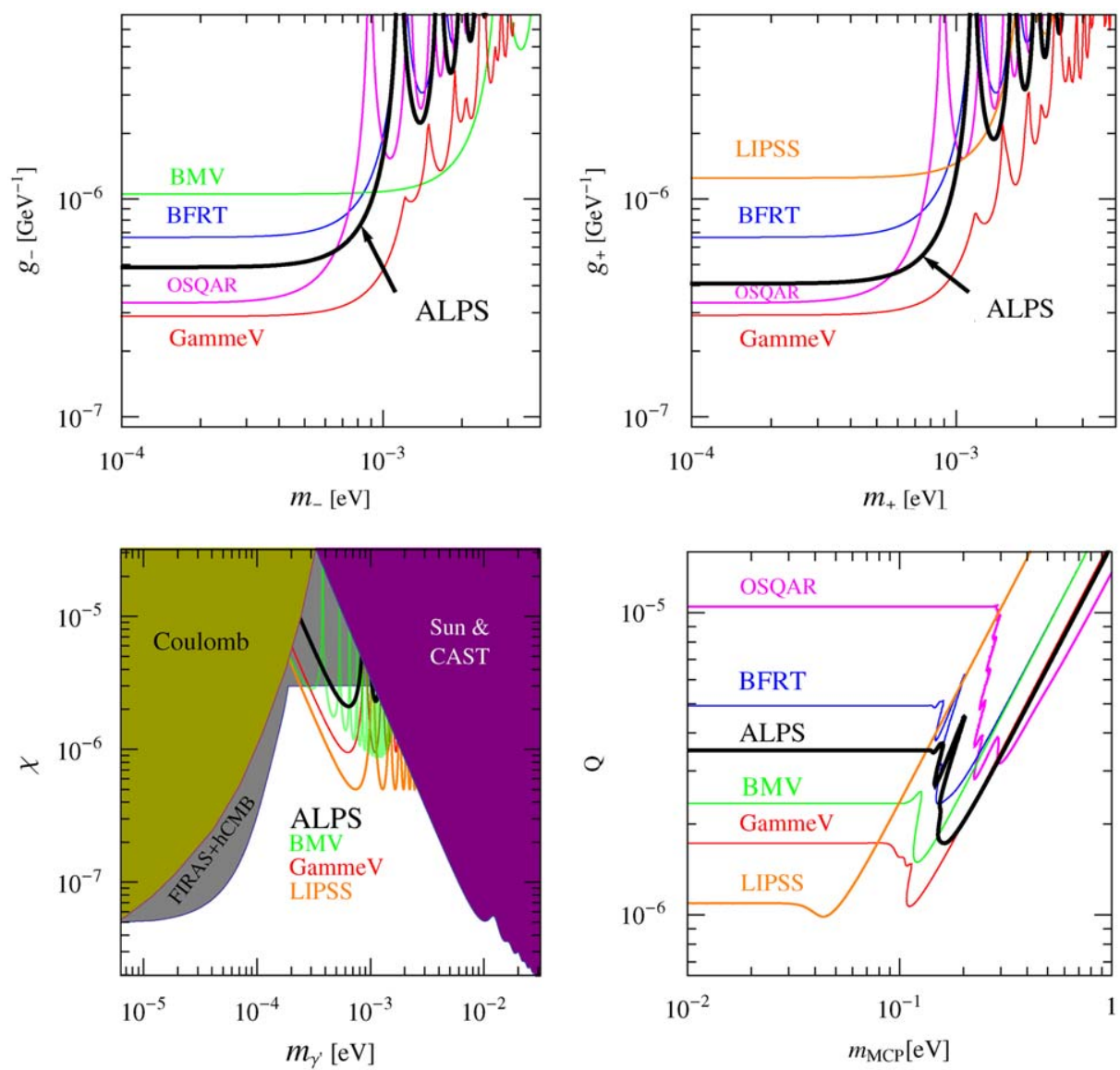

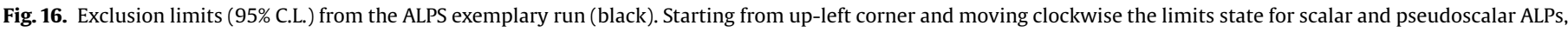

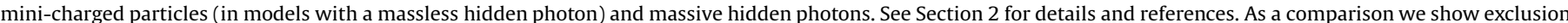

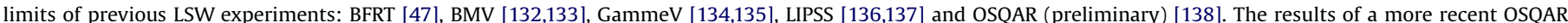

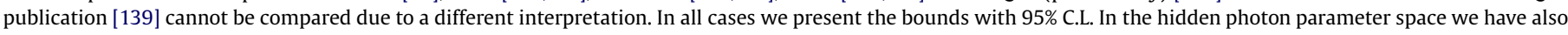

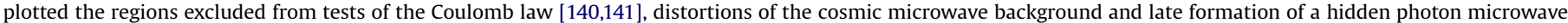
background [11], and emission of hidden photons from the Sun [4]. Similar exclusion bounds for the case of ALPs and MCPs are reviewed for instance in Refs. [2,19]. 
Table 5

Upper limits for the search for WISPs in the data presented in this paper.

\begin{tabular}{lll}
\hline WISP & $95 \%$ C.L. limit & Mass \\
\hline $\mathrm{ALP} \mathrm{0}^{-}$ & $g<4.9 \times 10^{-7} \mathrm{GeV}^{-1}$ & $m_{-} \lesssim 0.5 \mathrm{meV}$ \\
$\mathrm{ALP}^{+}$ & $g<4.1 \times 10^{-7} \mathrm{GeV}^{-1}$ & $m_{+} \lesssim 0.5 \mathrm{meV}$ \\
$\mathrm{HP}$ & $\chi<2.1 \times 10^{-6}$ & $m_{\gamma^{\prime}} \approx 0.7 \mathrm{meV}$ \\
$\mathrm{MCP}+\mathrm{HP}$ & $\mathrm{Q}_{\mathrm{MCP}}<3.5 \times 10^{-6}$ & $m_{\mathrm{MCP}} \lesssim 0.1 \mathrm{eV}$ \\
\hline
\end{tabular}

To derive upper limits for the WISP induced photon flux from our data we have to consider the quantum efficiency of the camera $(0.6 \pm 0.1)$, the electron/ADU conversion factor of the camera $(1.46 \pm 0.05)$ and the fraction of the signal contained in the search region $(0.75 \pm 0.15)$. Other effects like the light absorption in the window at the exit of the detector side vacuum tube and in the lens in front of the camera as well as the reflection at the $45^{\circ}$ mirror (see Fig. 3 ) are estimated to result in a light collection efficiency of $0.98 \pm 0.02$. This gives a flux limit of $29 \mathrm{mHz}$ at 95\% C.L. (see Table 3) using the method of Ref. [131]. Taking into account the effective flux of incoming photons from Table 2 one arrives at the conversion probabilities in Table 4.

For determining the limits on the photon-WISP mixing strength as a function of the WISP mass we use the experimental parameters summarized in Fig. 15.

The uncertainties of the magnetic field strength, $\sigma_{B}$, (when relevant, i.e. for ALPs and MCPs) and of the tube lengths, $\sigma_{L}$, are taken into account by a Monte Carlo calculation. Here we conservatively use $\sigma_{B}=0.01 \mathrm{~T}$ and $\sigma_{L}=0.1 \mathrm{~m}$. The results are shown in Fig. 16 and in Table 5.

Note that the LSW probability is the product of the photonWISP conversion probability and the WISP-photon reconversion (both given by (2)) which makes it proportional to the fourth power of the relevant coupling constant that drives the photonWISP conversions (i.e. $g_{+}$for ALPs, $\chi$ for hidden photons and $\varepsilon$ for MPCs). Ultimately this means that the sensitivity of such an experiment grows with only the fourth root of the available power. Still, as remarked in the introduction, the technology already at work in gravitational wave detectors can be used to obtain up to several tens of $\mathrm{kW}$ in the production cavity through essentially the same techniques explained in this paper. With only this improvement the ALPS experiment could surpass the sensitivities of the present LSW experiments covering large parts of unexplored WISP parameter space. Furthermore, the investment in development and characterization of resonant cavities in the ALPS experiment will eventually allow the set-up of a resonantly enhanced regeneration scheme [50-52] boosting the sensitivity much further.

\section{Conclusions}

The ALPS collaboration runs a "light shining through a wall" (LSW) experiment to search for photon oscillations into "weakly interacting sub-eV particles" (WISPs) inside of a superconducting HERA dipole magnet at the site of DESY.

In this paper we have described and characterized our apparatus and demonstrated the data analysis procedures. Our main result is the first successful integration of a large-scale optical resonator into a complete LSW experiment. This resonator serves as an amplifier for the photon flux in the production region of the experiment and thus boosts the experiment's sensitivity.

During a $31 \mathrm{~h}$ long exemplary run, the available laser light power to search for WISPs was increased by a factor of 43 . The upper limits on photon-WISP interactions derived from this brief run show that ALPS is very competitive with other state-of-the-art LSW experiments (see Fig. 16).

Moreover, we have identified the main limitations of our current set-up, showing that significant improvements, especially of the performance of the optical resonator, are possible. This opens a clear pathway for near-term future steps in increasing the sensitivity of the ALPS experiment.

\section{Acknowledgments}

The ALPS Collaboration thanks the MKS Group at DESY for support, especially H. Brück, E. Gadwinkel, C. Hagedorn, H. Herzog, K. Prüss and M. Stolper. Many thanks to U. Koetz for advice for selecting the camera and A. Zuber for the technical drawings. We acknowledge financial support from the Helmholtz Association and from the Centre for Quantum Engineering and Space-Time Research (QUEST).

\section{References}

[1] L.F. Abbott, P. Sikivie, Phys. Lett. B120 (1983) 133

[2] G.G. Raffelt, Stars as Laboratories for Fundamental Physics, The University of Chicago Press, Chicago, 1996, p. 664.

[3] G.G. Raffelt, Annu. Rev. Nucl. Part. Sci. 49 (1999) 163 hep-ph/9903472.

[4] J. Redondo, JCAP 0807 (2008) 008 arXiv:0801.1527 [hep-ph].

[5] S.N. Gninenko, J. Redondo, Phys. Lett. B664 (2008) 180 arXiv:0804.3736 [hep-ex].

[6] H. Georgi, P.H. Ginsparg, S.L. Glashow, Nature 306 (1983) 765.

[7] A. De Angelis, R. Pain, Mod. Phys. Lett. A17 (2002) 2491 arXiv:astro-ph/ 0205059 .

[8] A. Mirizzi, G.G. Raffelt, P.D. Serpico, Phys. Rev. D72 (2005) 023501 arXiv:astro-ph/0506078.

[9] A. Mirizzi, G.G. Raffelt, P.D. Serpico, Phys. Rev. D76 (2007) 023001 arXiv:0704.3044 [astro-ph].

[10] A. Mirizzi, J. Redondo, G. Sigl, JCAP 0903 (2009) 026 arXiv:0901.0014 [hepph].

[11] J. Jaeckel, J. Redondo, A. Ringwald, Phys. Rev. Lett. 101 (2008) 131801 arXiv:0804.4157 [astro-ph].

[12] A. De Angelis, O. Mansutti, M. Roncadelli, Phys. Rev. D76 (2007) 121301 arXiv:0707.4312 [astro-ph].

[13] J.-H. Huh, J.E. Kim, J.-C. Park, S.C. Park, Phys. Rev. D77 (2008) 123503 arXiv:0711.3528 [astro-ph].

[14] J. Isern, S. Catalan, E. Garcia-Berro, S. Torres, arXiv:0812.3043 [astro-ph].

[15] J. Isern, E. Garcia-Berro, S. Torres, S. Catalan, arXiv:0806.2807 [astro-ph].

[16] C. Burrage, A.-C. Davis, D.J. Shaw, arXiv:0902.2320 [astro-ph.CO].

[17] M. Fairbairn, T. Rashba, S. Troitsky, arXiv:0901.4085 [astro-ph.HE].

[18] M. Ahlers, arXiv:0904.0998 [hep-ph].

[19] J. Redondo, arXiv:0810.3200 [hep-ph].

[20] E. Masso, J. Redondo, JCAP 0509 (2005) 015 hep-ph/0504202.

[21] E. Masso, J. Redondo, Phys. Rev. Lett. 97 (2006) 151802 hep-ph/0606163.

[22] R.N. Mohapatra, S. Nasri, Phys. Rev. Lett. 98 (2007) 050402 arXiv:hep-ph/ 0610068.

[23] J. Jaeckel, E. Masso, J. Redondo, A. Ringwald, F. Takahashi, Phys. Rev. D75 (2007) 013004 hep-ph/0610203.

[24] P. Brax, C. van de Bruck, A.-C. Davis, Phys. Rev. Lett. 99 (2007) 121103 hep$\mathrm{ph} / 0703243$.

[25] D.D. Stancil, Phys. Rev. D76 (2007) 111701 arXiv:0704.0490 [hep-ph].

[26] J. Jaeckel, J. Redondo, A. Ringwald, arXiv:0903.5300 [hep-ph].

[27] S. Abel et al., The Physics Case for a Low Energy Frontier of Fundamental Physics, 2009, in preparation.

[28] E.G. Adelberger, J.H. Gundlach, B.R. Heckel, S. Hoedl, S. Schlamminger, Prog. Part. Nucl. Phys. 62 (2009) 102.

[29] R. Battesti, et al., in: Lecture Notes in Physics, vol. 741, 2008, pp. 199-237, arXiv:0705.0615 [hep-ex].

[30] S. Andriamonje, et al.CAST Collaboration, JCAP 0704 (2007) 010 hep-ex/ 0702006.

[31] E. Arik, et al.CAST Collaboration, JCAP 0902 (2009) 008 arXiv:0810.4482 [hep-ex].

[32] G. Cantatore, et al., CAST Collaboration, arXiv:0809.4581 [hep-ex]

[33] Y. Inoue, et al., Phys. Lett. B668 (2008) 93 arXiv:0806.2230 [astro-ph].

[34] H. Davoudiasl, P. Huber, JCAP 0808 (2008) 026 arXiv:0804.3543 [astro-ph].

[35] L.D. Duffy, et al.ADMX Collaboration, Phys. Rev. D74 (2006) 012006 arXiv:astro-ph/0603108.

[36] A.C. Melissinos, arXiv:0807.1092 [hep-ph]

[37] B. Dobrich, H. Gies, arXiv:0904.0216 [hep-ph].

[38] J. Jaeckel, A. Ringwald, Phys. Lett. B659 (2008) 509 arXiv:0707.2063 [hep-ph]. 
[39] J. Jaeckel, J. Redondo, Europhys. Lett. 84 (2008) 31002 arXiv:0806.1115 [hep-ph].

[40] H. Gies, J. Jaeckel, A. Ringwald, Phys. Rev. Lett. 97 (2006) 140402 arXiv:hepph/0607118.

[41] H. Gies, J. Jaeckel, A. Ringwald, Europhys. Lett. 76 (2006) 794 arXiv:hep-ph/ 0608238.

[42] H. Gies, J. Jaeckel, arXiv:0904.0609 [hep-ph].

[43] L.B. Okun, Sov. Phys. JETP 56 (1982) 502.

[44] A.A. Anselm, Yad. Fiz. 42 (1985) 1480.

[45] K. Van Bibber, N.R. Dagdeviren, S.E. Koonin, A. Kerman, H.N. Nelson, Phys. Rev. Lett. 59 (1987) 759.

[46] G. Ruoso, et al.BFRT Collaboration, Z. Phys. C56 (1992) 505.

[47] R. Cameron, et al.BFRT Collaboration, Phys. Rev. D47 (1993) 3707.

[48] H. Luck, et al., Class. Quant. Grav. 23 (2006) 71.

[49] LIGO Collaboration, arXiv:0711.3041v1 [gr-qc].

[50] F. Hoogeveen, T. Ziegenhagen, Nucl. Phys. B358 (1991) 3.

[51] P. Sikivie, D. Tanner, K. van Bibber, Phys. Rev. Lett. 172002 (2007).

[52] G. Mueller, P. Sikivie, D.B. Tanner, K. van Bibber, arXiv:0907.5387 [hep-ph].

[53] E. Zavattini, et al.PVLAS Collaboration, Phys. Rev. Lett. 96 (2006) 110406 hep-ex/0507107.

[54] E. Zavattini, et al.PVLAS Collaboration, Phys. Rev. D77 (2008) 032006 arXiv:0706.3419 [hep-ex].

[55] S.-J. Chen, H.-H. Mei, W.-T. Ni, Q \& A Collaboration, Mod. Phys. Lett. A22 (2007) 2815 arXiv:hep-ex/0611050.

[56] R. Battesti, et al.BMV Collaboration, Eur. Phys. J. D46 (2008) 323 arXiv:0710.1703vl [physics.optics].

[57] ALPS Collaboration, K. Ehret, et al., hep-ex/0702023.

[58] ALPS Collaboration, K. Ehret, arXiv:0812.3495 [hep-ex].

[59] C.T. Hill, G.G. Ross, Nucl. Phys. B311 (1988) 253.

[60] Y. Liao, Phys. Lett. B650 (2007) 257 arXiv:0704.1961 [hep-ph].

[61] J. Redondo, Can the PVLAS particle be compatible with the astrophysical bounds? Ph.D. Thesis, IFAE (UAB), 2008, arXiv:0807.4329 [hep-ph].

[62] Y. Nambu, G. Jona-Lasinio, Phys. Rev. 122 (1961) 345.

[63] J. Goldstone, A. Salam, S. Weinberg, Phys. Rev. 127 (1962) 965.

[64] S. Weinberg, Phys. Rev. Lett. 29 (1972) 1698.

[65] S. Weinberg, Phys. Rev. Lett. 40 (1978) 223.

[66] F. Wilczek, Phys. Rev. Lett. 40 (1978) 279.

[67] R.D. Peccei, H.R. Quinn, Phys. Rev. Lett. 38 (1977) 1440

[68] R.D. Peccei, H.R. Quinn, Phys. Rev. D16 (1977) 1791.

[69] M. Dine, W. Fischler, M. Srednicki, Phys. Lett. B104 (1981) 199.

[70] A.R. Zhitnitsky, Sov. J. Nucl. Phys. 31 (1980) 260.

[71] J.E. Kim, Phys. Rev. Lett. 43 (1979) 103.

[72] M.A. Shifman, A.I. Vainshtein, V.I. Zakharov, Nucl. Phys. B166 (1980) 493.

[73] L.M. Krauss, F. Wilczek, Phys. Lett. B173 (1986) 189.

[74] R.D. Peccei, T.T. Wu, T. Yanagida, Phys. Lett. B172 (1986) 435.

[75] F. Wilczek, Phys. Rev. Lett. 49 (1982) 1549.

[76] Y. Chikashige, R.N. Mohapatra, R.D. Peccei, Phys. Rev. Lett. 45 (1980) 1926.

[77] Y. Chikashige, R.N. Mohapatra, R.D. Peccei, Phys. Lett. B98 (1981) 265.

[78] E.J. Chun, J.E. Kim, H.P. Nilles, Nucl. Phys. B370 (1992) 105.

[79] A.E. Nelson, N. Seiberg, Nucl. Phys. B416 (1994) 46 arXiv:hep-ph/9309299.

[80] C. Coriano, N. Irges, E. Kiritsis, Nucl. Phys. B746 (2006) 77 arXiv:hep-ph/ 0510332.

[81] C. Coriano, N. Irges, Phys. Lett. B651 (2007) 298 arXiv:hep-ph/0612140.

[82] P. Svrcek, E. Witten, JHEP 06 (2006) 051 arXiv:hep-th/0605206.

[83] C. Wetterich, Nucl. Phys. B302 (1988) 668.

[84] P.J.E. Peebles, B. Ratra, Astrophys. J. 325 (1988) L17.

[85] J.A. Frieman, C.T. Hill, A. Stebbins, I. Waga, Phys. Rev. Lett. 75 (1995) 2077 arXiv:astro-ph/9505060.

[86] J. Khoury, A. Weltman, Phys. Rev. Lett. 93 (2004) 171104 astro-ph/0309300.

[87] A. Dupays, E. Masso, J. Redondo, C. Rizzo, Phys. Rev. Lett. 98 (2007) 131802 hep-ph/0610286.

[88] P. Fayet, Phys. Lett. B96 (1980) 83.

[89] P. Fayet, Nucl. Phys. B187 (1981) 184.

[90] R. Barbieri, S. Cecotti, Z. Phys. C33 (1986) 255.

[91] R. Foot, A.Y. Ignatiev, R.R. Volkas, Phys. Lett. B503 (2001) 355 astro-ph/ 0011156.

[92] E.C.G. Stueckelberg, Phys. Rev. 52 (1937) 41

[93] E.C.G. Stueckelberg, Helv. Phys. Acta 11 (1938) 225.

[94] B. Holdom, Phys. Lett. B166 (1986) 196.

[95] R. Foot, X.-G. He, Phys. Lett. B267 (1991) 509.

[96] K.R. Dienes, C.F. Kolda, J. March-Russell, Nucl. Phys. B492 (1997) 104-118 hep-ph/9610479.

[97] S.A. Abel, B.W. Schofield, Nucl. Phys. B685 (2004) 150 arXiv:hep-th/0311051.
[98] S.A. Abel, J. Jaeckel, V.V. Khoze, A. Ringwald, Phys. Lett. B666 (2008) 66 arXiv:hep-ph/0608248.

[99] S.A. Abel, M.D. Goodsell, J. Jaeckel, V.V. Khoze, A. Ringwald, JHEP 07 (2008) 124 arXiv:0803.1449 [hep-ph].

[100] D. Feldman, Z. Liu, P. Nath, Phys. Rev. D75 (2007) 115001 arXiv:hep-ph/ 0702123.

[101] M. Pospelov, A. Ritz, M.B. Voloshin, Phys. Lett. B662 (2008) 53 arXiv:0711.4866 [hep-ph].

[102] J. Redondo, M. Postma, JCAP 0902 (2009) 005 arXiv:0811.0326 [hep-ph].

[103] A. Ibarra, A. Ringwald, C. Weniger, JCAP 0901 (2009) 003 arXiv:0809.3196 [hep-ph].

[104] C.-R. Chen, F. Takahashi, T.T. Yanagida, Phys. Lett. B671 (2009) 71 arXiv:0809.0792 [hep-ph].

[105] N. Arkani-Hamed, D.P. Finkbeiner, T.R. Slatyer, N. Weiner, Phys. Rev. D79 (2009) 015014 arXiv:0810.0713 [hep-ph].

[106] D. Feldman, Z. Liu, P. Nath, Phys. Rev. D79 (2009) 063509 arXiv:0810.5762 [hep-ph]

[107] A. Ibarra, A. Ringwald, D. Tran, C. Weniger, arXiv:0903.3625 [hep-ph].

[108] B. Batell, M. Pospelov, A. Ritz, arXiv:0903.0363 [hep-ph].

[109] R. Essig, P. Schuster, N. Toro, Phys. Rev. D 80 (2009) 015003 [arXiv:0903.3941 [hep-ph]].

[110] R. Rahman, arXiv:0901.2111 [hep-ph].

[111] B. Batell, T. Gherghetta, Phys. Rev. D73 (2006) 045016 arXiv:hep-ph/ 0512356.

[112] M. Ahlers, H. Gies, J. Jaeckel, A. Ringwald, Phys. Rev. D75 (2007) 035011 arXiv:hep-ph/0612098.

[113] M. Ahlers, H. Gies, J. Jaeckel, J. Redondo, A. Ringwald, Phys. Rev. D76 (2007) 115005 arXiv:0706.2836 [hep-ph].

[114] G. Raffelt, L. Stodolsky, Phys. Rev. D37 (1988) 1237.

[115] C. Biggio, E. Masso, J. Redondo, Phys. Rev. D79 (2009) 015012 hep-ph/ 0604062.

[116] M. Frede, B. Schulz, R. Wilhelm, P. Kwee, F. Seifert, B. Willke, D. Kracht, Opt. Express 15 (2) (2007) 459.

[117] T. Kane, R. Byer, Opt. Lett. 10 (2) (1985) 65

[118] 〈http://www.sbig.com/sbwhtmls/online.htm〉.

[119] S.L. Adler, J. Gamboa, F. Mendez, J. Lopez-Sarrion, Ann. Phys. 323 (2008) 2851 arXiv:0801.4739 [hep-ph].

[120] G.D. Boyd, D.A. Kleinman, J. Appl. Phys. 39 (8) (1968) 3597.

[121] S. Spiekermann, F. Laurell, V. Pasiskevicius, H. Karlsson, I. Freitag, Appl. Phys. B 79 (2004) 211.

[122] S. Wang, V. Pasiskevicius, F. Laurell, J. Appl. Phys. 96 (4) (2004) 2023.

[123] K.H. Mess, P. Schmueser, S. Wolff, Superconducting Accelerator Magnets, World Scientific Publishing Co. Pte. Ltd., Singapore, 1996.

[124] A. Siegman, Lasers, University Science Books, 1986 (corrected corresponding to errata list published at $\langle$ http://www.stanford.edu/ siegman/lasers_book errata.pdf $>$ ).

[125] H. Kogelnik, T. Li, Appl. Opt. 5 (10) (1966) 1550.

[126] P. Kwee, F. Seifert, B. Willke, K. Danzmann, Rev. Sci. Instr. 78 (7) (2007) 073103.

[127] R. Pound, Rev. Sci. Instr. 17 (11) (1946) 490.

[128] R. Drever, J. Hall, F. Kowalsky, J. Hough, G. Ford, A. Munley, H. Ward, Appl. Phys. B 31 (2) (1983) 97.

[129] E. Black, Am. J. Phys. 69 (1) (2000) 79.

[130] M. Heurs, V. Quetschke, B. Willke, K. Danzmann, Opt. Lett. 29 (18) (2004) 2148.

[131] G. Feldman, R. Cousins, Phys. Rev. D (57) (1998) 3873.

[132] C. Robilliard, et al.BMV Collaboration, Phys. Rev. Lett. 99 (2007) 190403 arXiv:0707.1296 [hep-ex].

[133] M. Fouche, et al.BMV Collaboration, Phys. Rev. D78 (2008) 032013 arXiv:0808.2800 [hep-ex].

[134] A.S. Chou, et al.GammeV Collaboration, Phys. Rev. Lett. 100 (2008) 080402 arXiv:0710.3783 [hep-ex].

[135] M. Ahlers, H. Gies, J. Jaeckel, J. Redondo, A. Ringwald, Phys. Rev. D77 (2008) 095001 arXiv:0711.4991 [hep-ph].

[136] A. Afanasev, et al., LIPSS Collaboration, arXiv:0810.4189 [hep-ex].

[137] A. Afanasev, et al.LIPSS Collaboration, Phys. Rev. Lett. 101 (2008) 120401 arXiv:0806.2631 [hep-ex].

[138] R. Ballou, et al., OSQAR Collaboration, Summary of OSQAR First Achievements and Main Requests for 2008, CERN-SPSC-2007-039S; PSC-M-762, December 2007.

[139] P. Pugnat, et al.OSQAR Collaboration, Phys. Rev. D78 (2008) 092003 arXiv:0712.3362 [hep-ex].

[140] D.F. Bartlett, S. Loegl, Phys. Rev. Lett. 61 (1988) 2285.

[141] E.R. Williams, J.E. Faller, H.A. Hill, Phys. Rev. Lett. 26 (1971) 721. 